\title{
Stadtluft macht frei: Die Stadt in den Medienepochen der Gesellschaft
}

\author{
Von Dirk Baecker
}

Zusammenfassung: Der Beitrag untersucht die soziale Form der Stadt in den vier Medienepochen der Stammesgesellschaft (Mündlichkeit), antiken Gesellschaft (alphabetische Schrift), modernen Gesellschaft (Buchdruck) und nächsten Gesellschaft (der Computer und seine Derivate). Ausgehend von Max Webers These, dass die Stadt das Miteinanderleben der untereinander Unbekannten ermöglicht, untersucht der Beitrag die strukturelle und kulturelle Funktion der Stadt im Umgang mit dem „Überschusssinn“ (Niklas Luhmann) jedes neu auftretenden Verbreitungsmediums der Kommunikation. Als Einheit der Differenz dieser vier Medienepochen erweist sich die städtische Einrichtung von „Leerstellen“, die es erlauben, den Raum der Stadt als einen Raum der Vorbereitung von und des Umgangs mit Überraschungen zu strukturieren und zu kultivieren. In der „Stadt“ der Stammesgesellschaft sind diese Leerstellen die Orte des ,silent trade“, in der Stadt der Antike die oikoi, in denen die Privaten ihre Pläne schmieden, für deren Folgen sie auf der agorà Rede und Antwort stehen müssen, in der modernen Stadt die funktionalen Pläne, die laufend durchkreuzt werden, aber auch ständig nachgebessert werden können, und in der nächsten Stadt die posses, die das Gewebe der Stadt durchkreuzen und zerreißen, ohne ihm mehr als einen vorübergehenden Schaden zufügen zu können.

\section{I.}

Obwohl es soziologisch auf der Hand liegt, dass die Stadt ebenso sehr ein Produkt wie ein Träger des gesellschaftlichen Wandels, wenn nicht sogar, als Initiator von abweichenden Ereignissen, als Instanz struktureller Selektion und als Institution der Retention und Restabilisierung positiv und negativ selegierter Varianten, ein Produkt und Träger der sozialen Evolution ist (vgl. Campbell 1969; Luhmann 1997: Kap. 7), ist die Stadt vor allem als Struktur der Gleichzeitigkeit des Ungleichzeitigen auffällig. Nirgendwo sonst sind sowohl die lange Dauer als auch die flüchtige Gegenwart und der mutige Ausgriff in eine Zukunft, die vielleicht niemals kommen wird, so auffällig wie im Baubestand, im Grundriss, in den räumlichen Orientierungen, in den robusten Segregationen, in den Verteilungsmustern von Lebenschancen einer Stadt.

Diese Struktur der Gleichzeitigkeit des Ungleichzeitigen versuchen wir uns im Folgenden für eine Betrachtung der Stadt im Wandel der Medienepochen der Gesellschaft zunutze zu machen. Wir rechnen mit dem kundigen Blick des Städteplaners und Architekten für die Differenz von Infrastruktur und Oberflächenstruktur der Stadt, mit dem Gespür des Literaten für jene Orte der Stadt, an denen sich Lebensschicksale entscheiden oder eine neue Wendung nehmen können, ${ }^{1}$ und mit den besorgten Nachfragen jener Beobachter, die die Differenz von Stadt und Gesellschaft in den Blick nehmen, um Fallen, Risiken und Chancen der weiteren Entwicklung zu überprüfen (siehe zuletzt Burdett / Sudjic 2007). Ausgangspunkt ist immer

1 Siehe die Kriminalromane von Edgar Allen Poe und Raymond Chandler bis zu Andrew Vachss und Sara Paretsky und vgl. seine Vorbilder, die Romane von Charles Dickens, Joseph Conrad, Emile Zola, Fjodor Dostojewskij, Alfred Döblin und Upton Sinclair. Siehe auch Calvino (1985). Für das Interesse des Architekten und Städteplaners am literarischen Blick auf die Stadt plädiert auch Marcel Meili im Gespräch mit Jacques Herzog (Meili 2006: 138). 
wieder, dass die Stadt einen Unterschied macht. ${ }^{2}$ Sie nimmt gesellschaftliche Tendenzen auf und spiegelt sie wider, hält sie jedoch auch auf Abstand. ${ }^{3}$ Sie ist ein Labor für neue Entwicklungen inklusive der Beobachtung dieser Entwicklungen (Lange 2007), aber auch der Ort einer sich selbst organisierenden Lebenswelt, die schon viele Moden überstanden hat. Platon war vermutlich der erste Beobachter, der die Stadt als Ort der Unruhe beschrieb und zugleich und im Anschuss an die noch nicht vergessenen dörflichen Strukturen der Stammesgesellschaft als Adresse für Ordnungsmaßnahmen im Horizont von Bemühungen um Gerechtigkeit in Anspruch nahm (siehe Platon 2000; vgl. Baecker 2004 a; und siehe zur „Gerechtigkeit“ als regulativer Idee in dörflich-bäuerlichen Gesellschaften Foster 1965 und 1967). Seither überlagern sich in der Stadt empirische Wirklichkeit und normative Ansprüche.

Wir machen uns diese strukturelle und kulturelle Differenz der Stadt im Folgenden für eine spezifische These zunutze, die nach Entsprechungen zwischen den Medienepochen der Gesellschaft auf der einen Seite und Medienepochen der Stadt auf der anderen Seite sucht. Wir fragen danach, ob man von einer Stadt der Stammesgesellschaft, der Antike, der Moderne und der nächsten Gesellschaft in einem ähnlichen Sinne sprechen kann wie von der Gesellschaft der Stammesgesellschaft, der Antike, der Moderne und der nächsten Gesellschaft (siehe auch Baecker 2007 a). Noch deutlicher als für die Gesellschaft rechnen wir für die Stadt mit der Parallelität der sich übereinander legenden Medienepochen, das heißt strukturell mit medialen Umbrüchen, die ihre Zeit in Anspruch nehmen, und kulturell mit Rückgriffen auf frühere Medienepochen, um die Anforderungen der nächsten zu bewältigen, wie auch mit Vorgriffen auf spätere Medienepochen, um die Traditionen der früheren zu überwinden. Wir arbeiten an einer Kulturtheorie der Stadt, die diese als Ort des Austrags gesellschaftlicher Umbrüche beschreibt, wie auch an einer Kulturanalyse der Stadt, die die beiden Instrumente der Medienepoche und der Differenz der Medienepochen bereitstellt, um untersuchen zu können, um welche Umbrüche es sich handelt, wie sie sich auswirken und wie sie bewältigt werden.

Unsere Vermutung ist, dass die Gesellschaft ihre Stadt sowohl dafür in Anspruch nimmt, sich der Struktur und Kultur einer früheren Medienepoche zu vergewissern, als auch dafür, Orte und Räume auszuzeichnen, in denen sich bereits die Anforderungen der nächsten, der jeweils aktuellen Medienepoche beobachten und umsetzen lassen. Die Stadt soll Verstand und Gemüt der Menschen beruhigen und anregen (so Simmel 1995). Sie fasziniert dort, wo ihr beides gelingt.

\section{II.}

In der These von den Medienepochen der Stadt steckt eine Reihe von Vorannahmen, die wir benennen müssen, bevor wir damit beginnen können, die These zu veranschaulichen und zu überprüfen. Die erste Annahme ist die vom Singular der Institution Stadt. Wir gehen davon aus, dass es menschheits- und gesellschaftsgeschichtlich nur eine Stadt gibt, die in einer Fülle unterschiedlicher regionaler und historischer Formen immer wieder dieselbe Funktion erfüllt und daher auch immer wieder, mit welcher bezeichnenden Unschärfe auch immer, als Stadt erkennbar ist (siehe zur These eines relativ begrenzten Repertoires von Institutionen in jeder

2 Berking / Löw (2005) unterstreichen das Interesse der Soziologie an einer „Eigenlogik der Städte“, meinen damit jedoch, wie der Titel ihres Beitrags andeutet, eher eine Eigenlogik, die verschiedene Städte untereinander unterscheidet, nicht etwa einen Unterschied, der die Stadt als solche von anderen Orten des gesellschaftlichen Miteinanders unterscheidet (Berking / Löw 2005; siehe in diesem Sinne auch Löw 2008: insbes. 73ff). Peter Marcuse spricht ebenfalls von den Unterschieden, die eine Stadt intern nach Kulturen, Funktionen und Status strukturieren (Marcuse 2005), nicht jedoch von den Unterschieden, die man nur in der Stadt und nirgendwo sonst findet.

3 Vom Unterschied zwischen der lokalen DNA einer Stadt und der nationalen DNA einer Gesellschaft spricht Sudjic (2007: 47ff). 
Gesellschaft Malinowski 2005: 56 f). Diese Annahme macht allerdings nur Sinn, wenn man die Funktion der Stadt benennen kann. Die bloße Wiederholung des Namens ist noch kein Beweis. Der Name muss wiederholt werden, weil er in der Sache etwas Identisches trifft.

Unsere zweite Annahme ist im Anschluss daran, dass die Funktion der Stadt von Max Weber auf den Punkt gebracht worden ist. Die Stadt ist jener Ort, so lautet eine der Definitionen, für die Weber eine Schwäche hatte, in der die, ,sonst dem Nachbarverband spezifische, persönliche gegenseitige Bekanntschaft der Einwohner miteinander fehlt" (Weber 1990: 727; vgl. Baecker 2004 b). ${ }^{4}$ Die Funktion der Stadt besteht darin, das Miteinanderleben der Einwohner zu ermöglichen, obwohl, während und vielleicht sogar indem sie sich nicht kennen. Die Stadt abstrahiert von der persönlichen Bekanntschaft zugunsten eines Miteinanders, das anderen Regeln folgt als denen, die man befolgt, wenn man miteinander vertraut ist. Der Punkt ist für uns heute unscheinbar, weil selbstverständlich, aber soziologisch gesehen nicht nur deswegen brisant, weil die Soziologie sich grundsätzlich mehr für das Selbstverständliche als für das Auffällige interessiert (so mit großem Sinn für die methodologischen Schwierigkeiten, die daraus folgen, Malinowski 2005: 107), sondern vor allem deswegen, weil menschheitsgeschichtlich einiges dagegen spricht, dass solche Regeln des Miteinanders unter Unbekannten überhaupt entstehen konnten. Die Stammesgesellschaft schloss den friedlichen Verkehr mit Unbekannten nahezu vollständig aus - wir werden auf die für uns entscheidende Ausnahme gleich zu sprechen kommen. Wie also konnten Städte dann überhaupt entstehen? Überdies ist es nicht so, dass diese Unwahrscheinlichkeitsschwelle des friedlichen Verkehrs mit Unbekannten einmal genommen wurde und seither keinerlei Problem mehr darstellt. Sie kann jederzeit wieder sichtbar werden. Es genügt, dass wir in Situationen kommen, in denen die Stadt ihre Funktion aus welchen Gründen auch immer nicht erfüllt und wir mit einer quasi stammesgesellschaftlichen Struktur der Begegnung zwischen Unbekannten konfrontiert werden. ${ }^{5}$ Aber auch dann, wenn wir nicht an diesen Ernstfall denken, kann es für den soziologischen Blick nur verwunderlich sein, mit welcher Selbstverständlichkeit wir bei Leuten einkaufen, unsere Kinder von Leuten unterrichten lassen, uns von Leuten regieren, uns vor Gericht verurteilen oder auch verteidigen lassen oder uns von Leuten den wahren Glauben predigen lassen, die uns allesamt zunächst einmal unbekannt sind, so sehr wir dann auch im Einzelfall die Chance haben mögen, sie persönlich kennen zu lernen.

Max Weber jedenfalls konnte sich über diesen Umstand nicht genug wundern und hat aus dem Versuch seiner Erklärung eine Soziologie der Stadt entwickelt, die unter anderem den Monotheismus als jene Überwindung der Stammesreligionen beschreibt, die es ermöglichte, dass die einander Unbekannten in der Stadt eine kultische Gemeinschaft bilden konnten, indem sie denselben Gott anbeteten (Weber 1990: 746ff). Darüber hinaus waren die Differenzen zwischen denen, die in einem Stadtverbund zusammen kamen und nun aufeinander angewiesen waren, um sich gegen andere Städte oder gegen kirchliche und fürstliche Herrschaftsansprüche zu verteidigen, immer wieder sichtbar genug, um gleichsam als Gegenmittel Formen der städt-

4 Die ,urban anthropology“ arbeitet offenbar ohne Verweis auf Max Weber mit demselben Ausgangspunkt und akzentuiert die Anonymität der Stadt ebenso wie die Fluidität der Beziehungen, die sie ermöglicht. Siehe im Anschluss an Lyn H. Lofland und Colette Pétonnet: Lindner (2005: 59).

5 Das Verbrechen in der Stadt ist nicht nur deswegen faszinierend, weil es für Leib und Leben gefährlich, wenn nicht sogar tödlich ist, sondern auch deswegen, weil ihm diese archaische Qualität der Begegnung zwischen Unbekannten eignet, mit der es alle urbanen Errungenschaften unterläuft und dadurch bestätigt. Gegenüber dieser Faszination eines Jack the Ripper verblasst jede Statistik, die darauf hinweist, dass die überwältigende Anzahl aller Morde unter Angehörigen verübt wird. 
ischen Verschwörung unter deswegen so genannten Eidgenossen zu erfordern, die stark genug waren, diese Differenzen zu überbrücken. ${ }^{6}$

Halten wir als unsere zweite Annahme fest, dass es die soziale Funktion der Stadt ist, das Miteinanderleben von untereinander Unbekannten zu ermöglichen. Halten wir auch fest, dass wir noch nicht wirklich wissen, wie diese Funktion jeweils erfüllt wird. Wir beschreiben die Stadt als ein X, das diese Funktion erfüllt, ohne uns darauf festlegen zu müssen, ob dies an einer kultischen Gemeinschaft, an gemeinsamen wirtschaftlichen Interessen, an einem durchsetzungsfähigen politischen und rechtlichen Herrschaftsapparat, an einer soliden Fest- und Kneipenkultur, an sonntäglichen Promenaden, an örtlichen Sportvereinen oder an der städtischen Kulturpolitik liegt. All dies spielt eine Rolle, aber in unterschiedlicher Weise und in einer Gemengelage, die als lokale Eigenheit verstanden werden kann, die ihrerseits stilisiert werden kann und so einen eigenen Beitrag zur Erfüllung der Funktion leistet.

Die Stadt selber ist die soziale Form, in der einander Unbekannte miteinander leben. Überall dort, wo dies möglich ist, sprechen wir von einer Stadt. Diese Stadt lebt davon, Problem und Lösung des Problems, Norm und Abweichung von der Norm, eine Nachbarschaft des Miteinanders, Gegeneinanders, Nebeneinanders und Übereinanders zugleich zu sein. Daraus gewinnt sie die institutionelle Robustheit und Flexibilität, die historisch und regional an ihr so auffällig sind.

Die Stadt ist diese soziale Form aber nur dann, wenn sie auffälliger, das heißt zugänglicher und folgenreicher als andere soziale Formen Leerstellen einrichtet, die sichtbar und erlebbar noch nicht definiert sind, aber noch definiert werden können. ${ }^{7}$ Die Stadt inszeniert den Raum der Wahl, der Entscheidung, wen man aus welchem Anlass trifft oder nicht trifft, kann dies aber nur dann, wenn dieser Raum nur unspezifisch definiert ist. Die öffentlich zugänglichen Plätze, ja die Öffentlichkeit schlechthin sind dieser Raum ebenso wie die kultischen Plätze, möglicherweise die Religion schlechthin, die beide nicht etwa festschreiben, was auf diesen Plätzen geschieht, sondern stattdessen festschreiben, dass auf ihnen Entscheidungen getroffen werden können, die noch nicht getroffen sind. Das öffnet die Stadt für ein bewegliches Sach-, Zeit- und Sozialkalkül, angesichts dessen sich jeder jederzeit fragen kann, mit wem er sich wann worauf wie lange einlässt. ${ }^{8}$ Die Stadt spielt an dieser Stelle den Lärm gegen die Stille aus, den Marktplatz gegen den Kirchenraum, und rechnet damit, dass im Lärm und in der Stille

6 Max Weber (1990: 749), spricht von einer „,usurpatorischen Verbrüderung“, die man, hält man sich die Konkurrenz der Stadt mit dem Staat vor Augen, auch als eine Geschichte der Emanzipation lesen kann, so Siebel (2000: 264, und 2004: 13). Dass man hier nicht immer sicher sein kann, wer sich von wem emanzipiert, zeigt Charles Tilly, der im Hinblick auf die Konkurrenz zwischen Stadt und Staat um die Kontrolle über Prozesse der Kapitalakkumulation deswegen von „liaisons dangereuses“" (Tilly 1992: 58), spricht. Städte finanzieren, Staaten bewaffnen die stehenden Heere, deren Rolle in der Nationalisierung so unverzichtbar ist wie in der Industrialisierung.

7 Das gilt auch dann, so Kevin Lynch, wenn die Stadt andererseits darum bemüht sein muss, mithilfe von Wegen, Grenzlinien, Bereichen, Brennpunkten und Merkzeichen „lesbar“ zu werden (Lynch 2007: insbes. $15 \mathrm{f}$ und 60ff). Leerstellen versetzen den Beobachter in die aktive Rolle, sich sein Stadtbild selber konstruieren zu müssen, aber auch zu können. „Eine Landschaft, in der jeder Stein eine Geschichte erzählt, macht die Erfindung neuer Geschichten schwierig" (Lynch 2007: 16). Ich danke F. Pavel Pavelka, Wien, für den Hinweis auf die Studie von Kevin Lynch sowie auf die formtheoretische Brauchbarkeit dieser Studie.

8 Das ist einer der Gründe dafür, dass Harrison C. White (1995: 1054ff) vorschlagen konnte, die sozialphilosophisch immer etwas zu emphatisch gefasste Kategorie der „Öffentlichkeit“ soziologisch genauer als „switch“ zu fassen, als Stelle, an der man sich entscheiden kann, aber auch muss, was man anschließend tut. 
jeweils andere Übergänge zugunsten anderer Möglichkeiten zugänglich sind. ${ }^{9}$ Beide sind Orte der Entscheidung, auch wenn, das wird für die folgenden Überlegungen wesentlich sein, diese Entscheidung an diesen Orten weder vorbereitet noch getroffen wird.

Unsere dritte und letzte Annahme ist die Annahme einer heuristischen Fruchtbarkeit der Unterscheidung von vier Medienepochen der Gesellschaft (vgl. McLuhan 1994; Goody / Watt / Gough 1981; Ong 1987). Wir unterscheiden die Stammesgesellschaft in der Auseinandersetzung mit der Einführung der Sprache, die antike Hochkultur in der Auseinandersetzung mit der Einführung der Schrift, die moderne Gesellschaft in der Auseinandersetzung mit der Einführung des Buchdrucks und die nächste Gesellschaft in der Auseinandersetzung mit der Einführung des Computers und seiner Derivate. Die These ist historisch grob geschnitzt, weil sie zahlreiche Differenzen unter den Sprachen und Schriften menschlicher Gesellschaften ebenso vernachlässigt wie die sozialen Auswirkungen von Fotografie, Film und Fernsehen, aber sie erweist ihren heuristischen Wert darin, dass sie das Auftreten eines neuen Verbreitungsmediums der Kommunikation nicht primär als ökonomisch willkommene Senkung der Transaktionskosten menschlicher Verständigung interpretiert, sondern als Überforderung bis dato bewährter gesellschaftlicher Strukturen, die kulturell auf die neuen Möglichkeiten umgestellt werden müssen, wenn die Einführung des neuen Verbreitungsmediums gelingen soll (Luhmann 1997: 405ff; vgl. Baecker 2004 c, 2007 a, 2007 b und 2007 / 08). Wir können vor dem Hintergrund dieser Annahme von einer Strukturform der Gesellschaft sprechen, die darin besteht, die mit einem neuen Medium technisch mögliche Verbreitung von Kommunikation auch sozial sicherzustellen. Und wir können, mit zwei Begriffen von Niklas Luhmann, von einer „Kulturform“ der Gesellschaft sprechen, die es erlaubt, selektiv mit dem „Überschusssinn“ der Kommunikation in einem neuen Verbreitungsmedium umzugehen, ohne die strukturellen Möglichkeiten der Kommunikation in diesem Medium zu beschneiden (Luhmann 1997: 410 f; vgl. mit dem Begriff des „overflow“ Callon 1998).

Für jede Medienepoche der Gesellschaft kann man sich dann auf die Suche nach ihrer Strukturform und ihrer Kulturform machen. Und für jede Medienepoche der Gesellschaft kann man fragen, welche Form in ihr jene Stadt annimmt, die in jeder Gesellschaft dieselbe Funktion erfüllt, nämlich Unbekannte miteinander leben zu lassen.

\section{III.}

Dieser nicht ganz unbescheidene, weil immerhin einen großen Teil der Menschheitsgeschichte abdeckende, aber nicht zu anspruchsvolle, weil mit einer einzigen Problemstellung auskommende Apparat der Beobachtung und Beschreibung von Gesellschaft erlaubt es uns nun, uns zur Bestimmung der gegenwärtigen Situation der Stadt auf einen Überblick über die Geschichte der Stadt einzulassen, dem wir einen medienarchäologischen Charakter geben. ${ }^{10} \mathrm{Wir}$ gehen nicht davon aus, dass sich die Medienepochen der Gesellschaft und ihrer Stadt historiographisch scharf unterscheiden und voneinander abgrenzen lassen, sondern davon, dass sich die Medienepochen überschneiden und überlagern, so dass wir es gegenwärtig mit (mindestens) vier verschiedenen Unterformen der Form der Stadt zu tun haben. Denn in der aktuellen Gesellschaft im Übergang von der modernen zur nächsten Gesellschaft muss nicht nur der

9 Ich verdanke diese Idee einer Diskussion von Charles Eck von Schanzenbach und Anita Pöltl. Der berühmte Satz von Michel Serres, „Die Stadt macht den Lärm, aber der Lärm macht die Stadt" (Serres 1981: 29), müsste deswegen ergänzt werden: Die Stadt braucht die Stille, und die Stille braucht die Stadt. Lärm und Stille markieren Leerstellen, aber erst ihre Differenz markiert die Form, mit der die Stadt etwas anfangen kann. Denn auf dem Lande hört sich die Stille, wie Gavino Ledda in seinem Roman Padre Padrone zu erzählen weiß, ganz anders an: Sie knallt (Ledda 1980).

10 Siehe zu einem hierfür brauchbaren Begriff der Archäologie Foucault (1997) und mit dem Exempel einer Archäologie der Stadt Rom als Vorbild einer Analyse des Seelenlebens Freud (1994: 37 f). 
Überschusssinn der Kommunikation im Medium des Computers, sondern müssen auch und nach wie vor der Überschusssinn in den Medien des Buchdrucks, der Schrift und der Sprache bewältigt werden. Wir lassen nicht nur rechnen, wir surfen und emailen nicht nur, sondern wir drucken nach wie vor, wir lesen das Gedruckte, wir schreiben und lesen nach wie vor auch Handgeschriebenes und wir sprechen miteinander und hören uns zu. Das alles bleibt so riskant und überfordernd, wie es immer war. Miteinander zu reden und einander Briefe zu schreiben, wird nicht dadurch einfacher, dass man auch Tageszeitungen lesen und im Internet recherchieren kann. Im Gegenteil.

In der Stadt überlagern sich diese Medienpraktiken. Wir werden mit ihnen konfrontiert, wir benutzen sie, wir wechseln zwischen ihnen, zuweilen treffen wir eine strategische Auswahl zugunsten der einen und gegen eine andere. Und immer öfter greifen wir zum Handy, weswegen wir herausfinden müssen, wie dieses zu den vier Kommunikationsmedien steht, auf deren Betrachtung wir uns hier konzentrieren.

Michel de Certeau wirbt in seiner „Kunst des Handelns“ für eine Kunst des Spaziergangs zur Erforschung und Beschreibung der Stadt, deren Hauptmotiv es ist, unserer ,, unheimlichen Vertrautheit" mit der Stadt Raum zu geben, indem sie „Platz für die Leere“ schafft und dann beobachtet, welche Identitäten diese Leere zu besetzen suchen (de Certeau 1988: 187 und 192ff). ${ }^{11}$ Wir folgen dieser Empfehlung, indem wir zum einen behaupten, dass die Stadt strukturell und kulturell am besten verstanden und beschrieben ist, wenn man zeigen kann, wie sie zur Lösung ihres Problems wird, indem sie die Leere schafft, die sie füllen kann, und zum anderen der Vermutung Raum geben, dass die unheimliche Vertrautheit daraus resultiert, dass die Stadt in jeder einzelnen ihrer Medienepochen auf eine strukturelle Praxis der vorherigen Epoche zurückgreift, um den kulturellen Anforderungen der jeweils nachfolgenden Epoche gerecht werden zu können. „Unheimlich“ ist dies, weil die epochale Differenz auffällt, ohne, da die Theorie der Medienepochen fehlt, entsprechend in Rechnung gestellt werden zu können. Und ,vertraut“ ist es, weil man das einst Bekannte und Selbstverständliche tut, wenn auch in einem neuen, so noch nicht gewohnten Zusammenhang.

\section{IV.}

Von einer „Stadt“ in der Stammesgesellschaft zu reden, wäre kaum möglich, hätten wir uns nicht mithilfe unserer funktionalen Definition der Stadt von der Erwartung gelöst, eine Stadt beginne dort, wo die Anzahl der Einwohner, die Verdichtung ihres Wohnens und die Vielfalt und Intensität ihres Verkehrs untereinander bestimmte Schwellenwerte übersteigt. Unsere Definition der Stadt als Ort, an dem Unbekannte miteinander leben können, liefert eine Suchheuristik, die nicht erst in der antiken Hochkultur, der Geburtsstunde der Stadt im klassischen Sinne (Benevolo 2007), fündig wird, sondern bereits in der Stammesgesellschaft, also in jener Gesellschaft, in der schon gesprochen und gehört, aber noch nicht geschrieben und gelesen wird.

Nehmen wir hinzu, dass wir nach Plätzen für die Leere suchen, das heißt nach Orten, an denen etwas passieren kann, was als Nichterwartetes behandelt werden kann, um verschiedene Anschlüsse sowohl nahezulegen als auch offen zu halten, ist bereits erkennbar, was als Stadt in der Stammesgesellschaft verstanden werden kann. Die anthropologische Forschung kennt unter dem Namen des „silent trade“ eine frühe Form des Marktes, der außerhalb der Dörfer stattfand, indem man dort zu bestimmten, mehr oder minder genau festgelegten Zeiten Dinge - unter dem Gesichtspunkt des decoupling dieser Dinge aus ihren gewohnten sozialen Bezügen

11 Die Stadt ist auf der sozialen Ebene jener ,calcul des places en tant que vides“, den Lacan (1978: 344) für das Bewusstsein und seine Sprache und damit als Grundlage einer Methodologie der sciences conjecturales (im Gegensatz zu den sciences exactes) beschrieben hat. 
kann man bereits von „Waren“ sprechen (Marx 1980: 49ff); Sohn-Rethel 1978; Backhaus 1997) - niederlegte, die von „Fremden“, mit denen man jede Begegnung vermied (weil man sich ,anstecken“ konnte), zu anderen Zeiten aufgenommen und durch eigene auf den Platz gelegte Dinge „bezahlt“ wurden (Grierson 1980; vgl. Agnew 1986: 17ff). War das Gebotene zu wenig, ließen die anderen es liegen und zogen mit ihren Dingen wieder ab. War der Preis zu niedrig, lagen beim nächsten Mal entweder gar keine Dinge bereit oder in geringerer Anzahl. Die Rede von „Fremden“, unter denen diese Form des Tausches stattfand, ist dabei nicht unproblematisch, weil Fremde einen Sozialstatus einer weiteren Entwicklungsstufe markieren: Fremd ist man, wenn man einem anderen Stamm angehört, jedoch Gastfreundschaft genießt, außerhalb derer man wie gehabt auch vom vorherigen Gastgeber ausgeraubt und erschlagen werden kann (Grierson 1980: 31ff; vgl. Stichweh 1992). Vielleicht sollten wir in unserem Zusammenhang besser von „Anderen“ in einem ebenso neutralen wie vorsichtigen Sinne reden.

Vermutlich wird man für die Entstehung dieses Ansatzpunktes für die Ausdifferenzierung der Stadt in der Stammesgesellschaft eine gewisse Größenordnung des Stammes annehmen müssen. So weit und so unvollkommen ich die Literatur kenne, sind dazu keine Angaben überliefert, doch wird man davon ausgehen können, dass die Risiken des silent trade erst dann eingegangen werden, wenn der Stamm groß genug ist, um Einzelne zu ermutigen, ihr Glück in der Konkurrenz um Besitz und Prestige mit Ihresgleichen außerhalb der Stammesgrenzen zu suchen, und stabil genug, um nicht auf die Idee kommen zu müssen, solche individuellen Abenteuer und ihre unerwarteten Konsequenzen bereits im Ansatz verhindern zu müssen. Robin Dunbar vermutet auf der Grundlage von Hypothesen zur Koevolution von Gesellschaft, Sprache und Neocortex, dass eine Stammesgröße von etwa 150 Mitgliedern weder unterschritten noch überschritten werden darf, um diese beiden Eigenschaften der kritischen Masse und hinreichenden Stabilität aufweisen zu können (Dunbar 2004: 111ff).

Die Märkte des silent trade sind „Städte“ im funktional präzisen Sinne des Wortes. Wir haben es mit Begegnungen unter Unbekannten zu tun, die dennoch eine Form finden, miteinander - wenn auch nacheinander - auszukommen. Wir haben es mit einem leeren Platz zu tun, der als ein Außerhalb des Dorfes, aber auch der Wildnis markiert wird und an dem etwas ausprobiert werden kann, was sonst nirgendwo stattfinden kann. Es versteht sich, dass dieser leere Platz nicht etwa undefiniert irgendwo zu suchen ist, sondern mindestens so scharf markiert und abgegrenzt wird wie alle anderen Plätze in der von Grenzziehungen aller Art vielfältig durchzogenen Stammesgesellschaft (vgl. Lévi-Strauss 1978: 148ff; Turner 1969; Leach 1979). Man wird annehmen können, dass die Fähigkeit, leere Plätze markieren und abgrenzen zu können, von einer bereits hoch entwickelten Praxis der Grenzziehung abhängig ist, die nicht nur mit Innenseiten und Außenseiten umgehen kann, sondern auch gelernt hat, Außenseiten als Innenseiten von etwas anderem zu betrachten und daraus eine gewisse Unbestimmtheit zu gewinnen. Kennzeichnend für Stammesgesellschaften ist der Respekt vor Schwellen und anderen Formen des Übergangs (zum Beispiel auch zwischen Kind und Erwachsenem, Unverheiratet und Verheiratet, Krank und Gesund, Tot und Lebendig, auf der Jagd und im Dorf), so dass man durchaus damit rechnen kann, dass wir es in diesen Gesellschaften nicht nur mit in der Natur der Sache liegenden territorialen Abgrenzungen zu tun zu haben, sondern darüber hinaus mit Gestaltungsspielräumen im Hinblick auf die Frage, was wann als welche Natur welcher Sache anzusehen ist, und mit verschiedenen Faltungen dazu gehörender Territorien (Leach 1979: 45ff).

Vor allem jedoch kann man an diesen Städten der Stammesgesellschaft die strukturelle Differenz studieren, die die These dieses Artikels ist. Obwohl die Stammesgesellschaft diejenige Gesellschaft ist, die bereits strukturelle und kulturelle Antworten auf die Einführung der Sprache gefunden hat, rekurriert sie ausgerechnet dort, wo sich zumindest rückblickend die histo- 
rische Weiterentwicklung der Gesellschaft zur Ausdifferenzierung einer Tauschwirtschaft andeutet, auf buchstäblich schweigende Begegnungen zwischen Unbekannten beziehungsweise auf die ausschließlich physische Kontrolle der Bewegung von Dingen und Körpern im Raum.

Unter dem medientheoretischen Gesichtspunkt, mit dem wir hier arbeiten, kann das nicht verwundern. Die Sprache ist nicht das willkommene Instrument zur schnelleren und leichteren Bewältigung bisher zu schwieriger Fragen, so als könne man schon dann miteinander handeln, wenn man Preise nennen und über sie streiten kann. Sondern die Sprache konfrontiert mit Zeichen, Worten für etwas, die im Hinblick auf das, was sie bezeichnen, wie sie es bezeichnen, von wem sie verwendet werden dürfen und wann sie verwendet werden dürfen, umständlich geregelt werden müssen, bevor und während ihr Gebrauch frei gegeben werden kann (Luhmann 1997: 205ff). Die Sprache erleichtert die Kommunikation nicht, sondern sie erschwert sie; und erst in dieser Form ruft sie strukturelle und kulturelle Antworten auf den Plan, die dann allerdings eine höhere Komplexität der Gesellschaft erreichbar machen als zuvor. Wir sprechen vom Referenzüberschuss der Sprache, von der losen, aus keiner Sache der Welt es sei denn der Gesellschaft selber heraus zu motivierenden Kopplung zwischen Wort und Ding, die strukturell nur durch die Konventionalisierung des Sprachgebrauchs durch Stämme (die, so ist zu vermuten, jetzt erst entstehen; zuvor gab es nur Familien und Horden) und kulturell nur durch die Einführung von Grenzen, an denen man erkennen kann, wer mit wem wann worüber sprechen darf (und kann), bewältigt werden konnte.

Das heißt, man verfügt in der Stammesgesellschaft bereits über die Kulturtechnik der Sprache, arbeitet jedoch mit einer Form der Stadt, die strukturell an die Zeiten vor der Ausdifferenzierung der Stammesgesellschaft erinnert, als man noch nicht miteinander sprechen konnte, sondern Begegnungen und deren Koordination (inklusive ihrer Vermeidung) im Medium der wechselseitigen Wahrnehmung steuerte. Die Stadt, so lautet deswegen unsere Hypothese, ist auf eine Gesellschaft angewiesen, die ihr einen Medienschritt voraus ist. Oder umgekehrt: In der Stadt vergewissert sich die Gesellschaft eigener, noch unausgeschöpfter Möglichkeiten, indem sie sich bei einer Strukturform rückversichert, mit der sie in der Vergangenheit bereits hinreichende evolutionär bewährte Erfahrungen gemacht hat. Oder noch knapper: In der Stadt wird die Gesellschaft zur Geschichte ihrer selbst.

Eine allzu konservative Formel ist dies nur dann nicht, wenn man die Stadt und die Gesellschaft gleichermaßen in den Blick nimmt, auf ihre Differenz achtet und sich an unsere Ausgangsbeobachtung der Gleichzeitigkeit des Ungleichzeitigen erinnert.

\section{V.}

Stadtluft macht frei. Max Webers Topos von der Stadt als Ort der Emanzipation (von den Zwängen des Dorflebens, der Kirche und der Fürstenhöfe; vgl. Weber 1990: 727ff, und 1991: 270ff) hat darin sein Recht, dass die Stadt innerhalb der Markierung, die eine gesellschaftliche Struktur setzt, auf die andere Seite der Unterscheidung wechselt und genau das freigibt, was die Struktur regelt. Beobachtbar wird damit das Freigegebene, unbeobachtbar werden Motiv und Struktur der Freigabe. Wenn man mit George Spencer-Browns Konzept einer Form der Unterscheidung arbeitet, die beide Seiten der Unterscheidung, die Trennung der beiden Seiten und den durch diese drei Werte - (a) Innenseite der Unterscheidung, (b) Außenseite der Unterscheidung und (c) Trennung zwischen den beiden Seiten der Unterscheidung - hervorgebrachten Raum der Möglichkeiten umfasst (Spencer-Brown 1997), sieht man, dass die Stadt sich mit diesem Wechsel auf die andere Seite der Unterscheidung nicht außerhalb, sondern innerhalb der Struktur der Gesellschaft bewegt. Deswegen kann Platon innerhalb seines Entwurfs einer gerechten Stadt von beidem reden, von der abweichenden Empirie der griechischen Polis, von ihren Fernhandelskaufleuten, die nur um des Geldes willen unterwegs sind, von ihren Sängern und Dichtern, die ihre unterhaltsamen und schrecklichen Lügen verbreiten, von 
ihren Handwerkern, die reich geworden den falschen Ehrgeiz entwickeln, in die Klasse der Krieger aufgenommen zu werden, und von ihren Kriegern, die zu Beratern werden wollen, einerseits und von den Normen der gerechten Stadt, in der jeder einzelne den ihm angestammten Platz einnimmt und nur das tut, was diesem Platz angemessen ist, andererseits (Platon 2000: 368ff).

Die antike Stadt ist die Aufhebung der stammesgesellschaftlichen Kulturform der Grenze und die Setzung einer neuen Kulturform, die es jetzt, nach der Einführung der alphabetischen Schrift, nicht mehr nur mit dem Referenzüberschuss der Sprache, sondern zusätzlich mit dem Symbolüberschuss der Schrift zu tun hat. Die Geheimnisse der Stammesgesellschaft, die im mykenischen Griechenland noch ihre Geltung hatten, werden buchstäblich ans Licht gezerrt, indem die Götterfiguren aus ihren Kammern, ihren Arkana, befreit werden und auf der Agora im vollen Sonnenlicht den Blicken aller freigegeben werden (so Vernant 1982: 41ff). Hatte die Stammesgesellschaft sich mithilfe ihrer Redeverbote (Geheimnisse) der Grenzen des Sagbaren vergewissert und diese durch unsichtbare Geister markiert, so vergewissert sich die antike Hochkultur nach der Einführung der Schrift mithilfe der Anbetung sichtbarer Götter einer sozialen Gemeinschaft, die Angehörige unterschiedlicher Stämme, wenn auch unter den Bedingungen des immer wieder nachzuregulierenden Ausschlusses von bisher Unbekannten (die als „Fremde“ eingeschlossen sind), übergreift (so auch de Coulanges 19612: 144ff).

Der Markt bleibt verdächtig wie eh und je, wie man an der aristotelischen Unterscheidung zwischen einer Erwerbslehre, die auf die angemessene Versorgung eines Haushalts zielt, und einer Erwerbskunst, der es um den Erwerb von Geld um des Geldes willen geht, studieren kann (siehe Aristoteles 1981: 1256ff; und vgl. Polanyi 1979), doch kommt nun ein neuer, nicht minder verdächtiger Ort hinzu, das Haus, der Oikos, in dem die zunehmend Privaten sich auf schriftlich festgehaltene Erinnerungen (Quittungen, Wechsel, Einlagen) beziehen, die durch die alte Kulturtechnik des offenen Wortes unter Männern nicht mehr kontrolliert werden können, sondern ihrerseits eine Kontrollfunktion der Ermöglichung längerfristiger Planung in Politik, Wirtschaft, Erziehung und Organisation auszuüben beginnen. Diese Häuser werden jedoch jetzt durch eine Polis kontextuiert, die es erlaubt, den Markt in die Stadt einzuführen (und dadurch erst zur Stadt im gebräuchlichen Sinne des Wortes werden zu lassen), und die die Häuser als Orte des privatwirtschaftlichen Kalküls einer Auseinandersetzung mit dem Markt freisetzt und als Orte der Herkunft politisch denkender Bürger in die Polis einbindet zugleich.

Vielleicht kann man, über Max Weber hinausgehend und damit unsere funktionale Beschreibung der Stadt abrundend, sogar annehmen, dass die Struktur und Kultur der Stadt nicht nur darin bestehen, das Zusammenleben der untereinander Unbekannten zu ermöglichen, sondern auch und erst recht darin, die miteinander Lebenden einander unbekannt werden zu lassen, um in die Abstraktion von Vertrautheit und in die Regulierung der Unbekanntheit sowohl Normen einzuhängen, deren Sinn andernfalls nicht eingängig wäre (etwa zum Themenkomplex „Gastfreundschaft"), als auch das Interesse an Normverletzungen zu wecken, das erforderlich ist, um eine mit wachsender Komplexität ausgestattete Gesellschaft überlebensfähig zu machen. Die Stadt sucht ihre eigene Größe und Dichte nicht etwa, um ihre Redundanzchancen zu steigern, sondern vor allem, um sich zur Varietät zu befähigen. Dazu jedoch müssen in die Strukturen des Sozialen Spannungen eingebaut werden, die weder zu groß noch zu gering sein dürfen, um den geschlossenen Kreis der Stadt laufend zu öffnen, ohne ihn zu verlassen. Die Stadt könnte der Mechanismus sein, der aus ein und derselben Dichte Tendenzen zur Abweichung und zur Annäherung gleichermaßen gewinnt.

Im Hinblick auf die Frage der Größenordnung erlauben wir uns die Hypothese, dass die antike Stadt die alten Grenzen der Stammesgesellschaft von 150 Mitgliedern unter der Bedingung überschreiten kann, dass sie eine Mauer baut, die sowohl eine erhebliche Erleichterung in der Notwendigkeit der Verarbeitung des Unterschieds zwischen Innen und Außen mit sich 
bringt als auch und gebunden daran Ansatzpunkte für die Binnendifferenzierung der Stadt bietet, indem Nähe und Entfernung zum Zentrum ebenso unterschieden werden können wie Nähe und Entfernung zur Mauer. Außerhalb der Mauer stößt man auf Leichen und damit auf das eigene, nur allzu gewiss ungewisse Schicksal, wie Leontius erfahren muss, von dem Sokrates berichtet, dessen Bericht uns Platon überliefert (Platon 2000: 439 e; vgl. Jacobs 2008: 77); aber auf der Mauer kann man auch jene Sonntagsspaziergänge unternehmen, auf denen sich die Bürger ihrer Wirklichkeit vergewissern. Ähnlich wie bereits in der Stammesgesellschaft mit ihrer kritischen Mitgliederzahl von 150 Menschen kommt es auch bei der Mauer darauf an, sowohl die Unruhe außerhalb der Stadt (die Fremde) als auch die Unruhe innerhalb der Stadt (die Leute) erwarten, in Rechnung stellen und kontrollieren zu können. Die Mauer erlaubt es, nur die Dinge und Ereignisse in die Stadt hineinzulassen, die man hineinlassen möchte, direkt außerhalb der Mauer Stellen vorzusehen, an denen das weniger Willkommene dennoch stattfinden kann, und von den Zinnen der Mauer und ihrer Türme aus die Weite einigermaßen sicher nicht aus dem Blick zu verlieren. Und die Mauer erlaubt es, den Leuten innerhalb der Stadt ihre Verbundenheit, ihren wechselseitigen Schutz und damit ihre gegenseitigen Ansprüche vor Augen zu führen, ohne darauf unnötig viele Worte verlieren zu müssen. Diese Evidenzleistungen der Mauer, zusätzlich unterstützt von der kollektiven Leistung ihres Baus, ihres Unterhalts und ihrer Ausstattung mit Gerät und Personal, tragen seither die strukturelle und kulturelle Annahme, dass man den Schritt vom Dorf zur Stadt macht, indem man eine Mauer zieht.

In der antiken Stadt zieht sich die Kommunikation zurück auf die Agora, um innerhalb eines nun Polis genannten Rahmens der Verständigung auf angemessene Ziele (teloi) zu regeln, welche Handlungen der Privaten man hinzunehmen bereit ist und welche nicht. Und es entstehen Wirtschaftslehren, die die in der Polis gefundene Vernunft den Oikoi schmackhaft zu machen versuchen (interessanterweise: durch die Wiedereinführung der Differenz zwischen Oikos und Polis in den Oikos in der Gestalt von reichem Mann und junger Ehefrau; so maßgebend Xenophon 1956), doch allen Beteiligten ist klar und Tragödien und Komödien führen es im Theater mit Lust und Faszination immer wieder vor, dass die Handlungen der Privaten im Haus und dann auch auf der Straße und auf den Fernhandelswegen zwischen den Städten auf die Normen der Polis nur Rücksicht nehmen, um sich auf neue Ideen bringen zu lassen.

Markt und Häuser sind nun gleichermaßen Plätze für die Leere, das heißt für immer wieder neue Setzungen von Identitäten in Anspruch zu nehmen, deren Überschuss freilich immer wieder auf das reduziert wird, was die Kommunikation derselben Gesellschaft nach ihren Regeln zu reproduzieren in der Lage ist. ${ }^{12}$ Sichtbar und Gegenstand vielfältiger Beobachtungen und Erzählungen wird das Kalkül der Privaten, unsichtbar, weil versteckt hinter Klugheitsregeln im Umgang mit Schicksalsfragen, bleibt die politische Ökonomie nicht zuletzt im Umgang mit Fragen der Vorsorge, denen dieses Kalkül zu folgen lernt (siehe exemplarisch und paradigmatisch den Verweis auf Sophrosyne, die Kunst der Selbstbeherrschung, am Schluss von Xenophons sokratischem Dialog über den Oikonomikos, Xenophon 1956, XXI, 12). In der antiken Stadt sind nicht nur die schweigenden Begegnungen freigegeben, sondern, wenn man so will, auch die redenden. Die Interaktion unter Anwesenden, in der tribalen Gesellschaft der streng zu regulierende Ernstfall, bekommt in der antiken Gesellschaft den Charakter des Ungebundenen, des Varianten und Diversen und kann in dieser Form nicht nur für strategische Verabredungen der Privaten genutzt werden, sondern auch für die demokratischen Absprachen

12 Hans Paul Bahrdt bindet daher die Urbanität und Humanität einer Ansiedlung unter Unbekannten an eine gut ausgebildete Polarität von Privatheit und Öffentlichkeit (Bahrdt 1998: 83 f). Beide Pole sind erforderlich, damit beide strapaziert werden können. Das Private muss dem Verdacht des Illegitimen, ja Verwerflichen ausgesetzt werden können, das Öffentliche als ebenso attraktiv wie folgenlos gelten dürfen, sonst haben wir es nicht mit einer Stadt, sondern mit einer Ideologie zu tun (siehe dazu auch Herlyn 2004). 
der „Oikosdespoten“ (Habermas 1990: 56; vgl. Brunner 1950: 122) auf der Agora, für das Spiel des Theaters und nicht zuletzt für eine neue Form der Bearbeitung von Sinnüberschüssen, die sich Philosophie nennt und in eigens dafür eingerichteten Akademien betrieben wird.

Die die antike Gesellschaft regulierende Kulturform ist nicht mehr die Grenze, sondern das Telos (,denn Ziel ist Grenze“, so Aristoteles 1970: 994 b), die Vorstellung des angemessenen Platzes, die wie einst die Grenze für beides Sinn hat, für die Erfüllung der Norm und für die Abweichung von der Norm. Sie gibt nicht nur den Blick, wie bereits in der Stammesgesellschaft, sondern auch die Rede frei und setzt stattdessen auf die Kontrolle der schriftlichen Kommunikation mithilfe der schriftlichen Kommunikation. Für die antike Stadt bedeutet das den Wechsel von den Grenzziehungen der tribalen Stadt (in der Form der Markierung nicht, also doch, zum Dorf gehörender Plätze des Tauschens) zur Symbolisierung institutioneller Zwecksetzungen, die man versucht, in Verfassungslehren festzuhalten und zu begründen. Ein Teil des Marktes kann nun in die dadurch im heutigen Sinne zur Stadt werdenden Stadt hineingeholt werden (bis heute gibt es gezähmte und ungezähmte Märkte, von denen erstere innerhalb der „Tore“ der Stadt und letztere außerhalb, „offshore“, abgehalten werden) und durch die markanten Gebäude der Tempel, Rathäuser, Gerichte und Schulen umgeben werden, die für die Augen von jedermann sichtbar machen, an welche Normen man sich zu halten gedenkt, während die eigentliche Stadt, die Einheit der Differenz von Normbefolgung und Normabweichung, in der die Reproduktionsfrage der Stadt und ihrer Gesellschaft den Blicken aller ausgesetzt wäre, nach wie vor nur strukturell, nicht kulturell begriffen wird, das heißt praktisch, aber nicht theoretisch realisiert und reflektiert wird.

\section{VI.}

Die historische und regionale Vielfalt, in der diese Entwicklung der Stadt sich vollzieht, macht es fast unmöglich, von der Stadt und ihrer Entwicklung jeweils im Singular zu reden. Wir tun es trotzdem, weil wir andernfalls den Anspruch auf ,Theorie“ aufgeben müssten und so keinerlei Möglichkeit mehr hätten, angesichts dieser Vielfalt zu erklären, warum wir dennoch und spontan leichter als reflektiert das Phänomen der Stadt von anderen Phänomenen zu unterscheiden in der Lage sind. Die spontane Leichtigkeit erklärt sich im Übrigen daraus, dass wir in ihr Unterscheidungen handelnd und erlebend mitvollziehen können, die wir auf der Ebene eines expliziten Wissens nicht zu kennen brauchen (Polanyi 1985), die reflektierten Schwierigkeiten dementsprechend daraus, dass die Explizierung dieses Wissens nur um den Preis seiner Fragwürdigkeit zu haben ist: Eine selbstverständlich gehandhabte Unterscheidung verliert ihre Selbstverständlichkeit, wenn wir sie auf ihre Form hin beobachten und dabei nicht umhin kommen, die beiden Seiten der Unterscheidung nicht etwa als Voraussetzung, sondern als Produkt der Unterscheidung zu entdecken. Wir bewegen uns nicht mehr in einer ontologisch abgesicherten, kategorial zu sortierenden Welt, sondern in einer ontogenetischen Welt, in einem konstruktivistisch reflektierten kognitiven Algorithmus, der alle Erkenntnis, die er produziert, den eigenen Operationen verdankt.

Es ist interessant, dass die antike Stadt der Geburtsort einer kategorial arbeitenden Ontologie ist. Mit dem Auftauchen der alphabetischen Schrift wird es nicht nur attraktiv, sondern auch notwendig, Menschen und Dingen Namen zu geben und sie verschiedenen Gattungen zuzuweisen, weil nur so die Norm und die Abweichung von der Norm hinreichend konkretisiert und feingesteuert werden können. Die Schichtenordnung, die sich die aristokratische Sklavenhaltergesellschaft der Antike gibt, wäre vermutlich kaum durchhaltbar, könnte man die Individuen nicht Familien und, sobald Herrschaftsansprüche erhoben werden, Dynastien zuordnen, die sowohl den normativen Anspruch deutlich machen, unter den sie sich stellen, als auch jedes Verständnis für individuell unvermeidbare Abweichungen aufbringen. Der Name überlebt das Fehlverhalten des Einzelnen nicht unbeschadet, aber er überlebt es. 
Deswegen werden Eigennamen in der antiken Gesellschaft und in ihrer Stadt so wichtig. Sie wiederholen die schriftliche Regulierung und schärfen den Sinn für die Vielfalt der Handlungen, die unter sie fallen (siehe die darauf reagierenden philosophischen Bemühungen bei Kripke 1980; und vgl. zur Funktion von Eigennamen in Netzwerken Heimer 1992). Eigennamen sind Realabstraktionen, die auf eine unbeobachtbare Wirklichkeit verweisen, die von der beobachtbaren nicht etwa widerlegt, sondern immer aufs Neue bestätigt wird.

Die moderne Stadt setzt hier, am Eigennamen als Realabstraktion, ihren Hebel an. Mit der Einführung des Buchdrucks tritt als ein neues Problem der Struktur und Kultur der Gesellschaft die Verbreitung und Selektion massenhaft vervielfältigter Kommunikation auf. In Flugblättern, Zeitungen, Büchern, Zeugnissen und Geldscheinen tritt eine Kommunikation auf, die aristotelisch nicht mehr gebändigt werden kann. Der strategische und taktische Spielraum für die Behauptung und Inszenierung von vorgeblich institutionell abgesicherten Teloi wird so sichtbar, dass die Teloi einer nach dem anderem mit den Mitteln der Vernunft, die ihre eigene Dekonstruktion zunächst noch vor sich hat, kritisiert und aufgeklärt, das heißt in kontingente Interessen übersetzt werden. Die moderne Gesellschaft ist durch einen Kritiküberschuss gekennzeichnet, ${ }^{13}$ nicht mehr nur durch einen Referenzüberschuss und einen Symbolüberschuss, und fraglich wird, welche Strukturen diesen Kritiküberschuss tragen und welche Kulturform ihn auf ein bewältigbares Maß reduziert und so ihrerseits reproduziert.

Die moderne Stadt jedenfalls ordnet sich nicht mehr nur wie noch die tribale Stadt durch den Ausschluss und die antike Stadt durch die Institution, sondern sie ordnet sich durch den Plan beziehungsweise durch die Form des Plans, das heißt durch die Unterscheidung des Planbaren vom Nichtplanbaren (vgl. Lefèbvre 1976). Dieser Plan setzt an den Eigennamen von Plätzen und Straßen, Firmen und Familien, Organisationen und Parteien an, nur um festzustellen, dass aus diesen Eigennamen nicht zwangsläufig folgt, welche Interessen sie haben, welchen Intentionen sie folgen und welche Potentiale sie entwickeln und verlieren.

Die Pläne erlauben es, die politische Reduktion der Stadt auf die Polis zu sprengen und sie in der Auseinandersetzung mit anderen Städten und mit Staaten auf ein zunehmend nationales Umfeld zu beziehen, das als Ressource ebenso wie als Bedrohung wahrgenommen wird (Tilly 1992). Ebenso wie Märkte und Häuser, die eingrenzen und beschränken zu können scheinen, was auf ihnen und in ihnen möglich ist, sollen auch Pläne festhalten, welche Entwicklung der Stadt sinnvoll scheint und welche nicht. Ebenso wie Märkte und Häuser jedoch, auf denen und in denen Überraschendes und Nichtintendiertes schon deswegen geschieht, weil ihr Möglichkeitenraum an denkbaren Handlungen, ihr Sinnüberschuss an möglichen Interpretationen durch die Eingrenzung allererst produziert werden, ist auch der Plan nicht auf die Exekution seiner selbst zu reduzieren. Mehr und mehr macht er sichtbar, was sich ihm nicht fügt. Mehr und mehr wird er zum Projekt seiner selbst, das nur noch in die Welt gesetzt wird, um Nachregulierungen zu ermöglichen, von denen man vorab nicht gewusst hätte, woran sie ansetzen und wie sie gerechtfertigt werden können.

Die Entdeckung der Differenz von Planbar und Nichtplanbar bekommt den Namen der Komplexität. Als komplex gilt, was geordnet ist, ohne geordnet werden zu können. Als komplex gilt, was sich aus der Besetzung von Leerstellen formiert, von denen man immer nur im Nachhinein sieht, warum man sie nicht prognostizieren konnte. ${ }^{14}$

13 Spätestens seit Immanuel Kant läuft der Kritiküberschuss positiv unter dem Namen der „Aufklärung““ (Kant 1964). Kant versuchte den Kritiküberschuss dadurch zu zähmen, dass der öffentliche Gebrauch der Vernunft Gelehrten anvertraut wurde, die sich ihrerseits vor einem Publikum zu verantworten hatten. Der Buchdruck ging über diesen Vorschlag hinweg.

14 Komplexität ist daher seit Weaver (1948) nur ein anderer Name für Selbstorganisation, so schwer es auch fällt, diese Erkenntnis immer wieder neu erst einzuholen, siehe jüngst Mitchell (2008). 
Mit den Plänen wird eine neue Größenordnung der Stadt erreicht. Für die Identität und Kontrolle ihrer Ereignisse ist sie jetzt nicht mehr an die 150 Mitglieder eines Stammes und auch nicht mehr an die Mauern der antiken Stadt, sondern an die Leistungsfähigkeit einer an ein bestimmtes Territorium gebundenen administrativen Einheit gebunden. Die moderne Stadt ist genau so groß, dass sie zum einen planen kann, was sie planen möchte, und zum anderen beobachten und bewältigen kann, was ihre Pläne durchkreuzt. Das ergibt keine wirklich stabile Einheit, so dass die administrativen Grenzen relativ häufig, denkt man an die etwas dauerhaftere Einrichtung einer Mauer, neu gezogen werden und damit auch die alte Stadt/Land-Differenz in eine neue Beweglichkeit bringen (Sieverts 1999). Hinzu kommt, dass die administrative Einheit der Stadt nur in einer laufenden Auseinandersetzung mit den administrativen Einheiten des Staates und seiner Länder festgestellt werden kann, so dass sich zum einen neue Interdependenzen zwischen Stadt und Nation ergeben, zum anderen jedoch der urbane Raum über alles hinausreicht, was sich ehemals als Stadt/Land-Differenz behaupten ließ (siehe hierzu zum Beispiel das urbane Bild der Schweiz in Diener et al. 2006). Die Stadt verliert an räumlicher Evidenz, aber nicht, das ist unsere These, an funktionaler Einheit und Notwendigkeit (siehe hierzu skeptischer Stichweh 1998: 353ff; und vgl. zur landschaftlichen Physiognomie einer durch den Verbrauch fossiler Energien und Massenmotorisierung gekennzeichneten Industriegesellschaft Sieferle 1997).

Mit jeder neuen Form, hervorgebracht durch eine neue Medienepoche, erhalten die alten Problemstellen eine beobachtbare Ordnungsfunktion in einer nach wie vor unbeobachtbar bleibenden Gesamtordnung. Nichts ordnet die moderne Stadt mehr, so die Chicagoer Stadtsoziologie, als das Wissen um ,neighborhoods“ und „,markets“, die durch hin und her eilende „news“ verknüpft und durch den Verweis auf „professions“ mit einem Sinn für die eigene Ordnung ausgestattet werden (Park 1967). Emile Durkheim konnte deswegen auf die Idee kommen, ein Bewusstsein für die nicht mehr in der Spitze einer Hierarchie zu repräsentierende Einheit der modernen Gesellschaft stattdessen an die Einsicht der Professionen beziehungsweise „Korporationen“ in die Gemeinsamkeit ihres Zusammenhangs zu binden (Durkheim 1988: Vorwort zur 2. Aufl.). Aber dafür ist es zu spät. Die Einheit der modernen Gesellschaft ist nur noch ökologisch zu denken und auch nur noch ökologisch zu praktizieren, wenn „Ökologie" heißen darf, dass nachbarschaftliche Beziehungen unterschiedlicher Art zu einer Verlässlichkeit finden, die in keinem Supersystem verankert werden muss, auch wenn es nach wie vor möglich ist, Symbole für sie zu finden, vom Kirchenbesuch über den Karneval bis zur Sportveranstaltung, in denen die Verlässlichkeit dieser Nachbarschaft gefeiert werden kann.

Die moderne Stadt jedenfalls kann nicht mehr über Gattungen und deren kategoriale Ordnung, sie kann nur noch funktional definiert werden (so etwa auch Frey / Zimmer 2001: 29, mit dem Begriff der functional community area). Le Corbusier sah dies in jedem Ozeandampfer gelungener verwirklicht als in der Architektur der zeitgenössischen Stadt, deren allzu konventionelle Einfälle, so seine Kritik, sich an Bildern ,sich schnäbelnder Tauben“ orientieren (so zehn Jahre nach dem Untergang der Titanic Le Corbusier 1963: 78 f, im Original 1922). Der Ozeandampfer ist die Komplexität der Stadt, verwandelt in den „Palast“ (Le Corbusier) der Feier ihrer selbst. Eine funktionale Definition bedeutet, dass die Namen der Dinge und Menschen, ihrer Organisationen und Pläne, zu Ansatzpunkten für neue Pläne werden, die sowohl mit ihrer Durchkreuzung als auch mit ihrer unsichtbaren Realisierung hinter dem Rücken der Akteure rechnen. Der Plan ist die Leerstelle, die als Ort der Begegnung unvorhersehbarer, aber wieder erkennbarer Spielzüge aller Beteiligten inklusive der Obdachlosen und Kriminellen, der Unternehmer und Politiker, der Prediger und Künstler gedacht werden kann.

In der modernen Stadt kann nicht nur das Reden, sondern auch das Schreiben und Lesen freigegeben werden. Das ist die Geburtsstunde des Intellektuellen, wie wir ihn kennen, ungebunden durch ältere Rücksichten auf die Kirche und den Staat. Die Kontrolle übernimmt der 
Buchdruck selbst, das heißt die Redundanz der Massenproduktion der Schriften, gesteuert durch das ein Mindestmaß an Varianz erzeugende Aktenwesen des Staates und der Industrie (unter diesem Aspekt wäre auch die Bürokratie neu zu würdigen, siehe nur Stinchcombe 2001). Die kritische Theorie sprach von einer durch die Kulturindustrie verwalteten Welt, mehr schlecht als recht bei Laune gehalten durch die Manipulationen der Massenmedien (Horkheimer / Adorno 1969: 128ff; und vgl. Baudrillard 1972), und wusste nicht, dass nur der Buchdruck dahinter steckt, der die Gesellschaft so hochgradig dynamisiert, dass ihr nur das Gleichgewicht der Indifferenz noch einen gewissen Halt geben kann.

Dieses Gleichgewicht der Indifferenz ist jedoch nicht der kulturkritisch anzunehmende Sündenfall, sondern selbst eine kulturelle Leistung, weil es Identität im Wechsel der Situationen, der Absichten, der Stimmungen und der Kritik schafft und weil es den empfindlichen und empfänglichen selektiven Zugriff auf Einzelnes nicht etwa ausschließt, sondern erst möglich macht (siehe zur Beschreibung von „Blasiertheit“, heute „coolness“, Simmel 1995; und vgl. Benjamin 1961, 2001).

Die Idee des Gleichgewichts, philosophisch von René Descartes („,cogito, ergo sum“ - egal wer mich kritisiert) und praktisch von der klassischen Ökonomie (im Sinne von Jean Baptiste Say: jedes Angebot schafft sich seine Nachfrage) auf den Punkt gebracht, ist die Kulturform einer modernen Gesellschaft, deren Städte durch nichts mehr gekennzeichnet sind als durch die Mobilität von und den Verkehr zwischen Leerstellen, an denen dann doch, sobald sie besetzt werden, nichts anderes passiert als das, was dort immer schon passiert ist.

Aber auch hier ist interessant, wie das Unbekannte nicht nur hingenommen, sondern auch produziert wird. Funktionale Beschreibungen sind so unzuverlässig in der Produktion von Identitäten wie Märkte und Häuser. Jede neue Abweichung von der Problemstellung wie von ihrer Lösung produziert eine wie immer minimale Überraschung, die schließlich nur noch in einer Gesellschaftsbeschreibung aufgefangen werden kann, die sich liberal nennt, weil sie den Spielraum anerkennt $u$ nd zuversichtlich ist, wieder einfangen zu können, was in ihm geschieht. Selten war das Unbekannte so domestiziert (siehe zum weder gesellschaftlich noch kulturell alternativlosen robusten Naturvertrauen der unternehmerischen Expansionisten Douglas 1989: $91 \mathrm{f})$. Tatsächlich ist die moderne Stadt, die mit ihrer funktionalen Selbstbeschreibung zugleich die Annahme produziert, sie sei sich selbst bekannt, der Ausgangspunkt für eine zuvor unvorstellbare Entdeckung und Eroberung der Welt als eines Raumes unbekannter, aber beherrschbarer Möglichkeiten. Den minimalen individuellen Abweichungen, an denen wie an einem seidenen Faden sie soziale Funktion der Stadt in dieser Zeit hängt, korrespondiert eine Beschreibung des Exotischen und Orientalischen, die das Unbekannte außerhalb der Mauern der modernen Stadt und ihrer westlichen Gesellschaft platziert und dort zum Gegenstand kolonialisierender und imperialistischer Maßnahmen macht.

\section{VII.}

Was wird sich in der nächsten Gesellschaft und ihren Städten ändern? Wie schon bei der tribalen und bei der antiken Stadt hat man auch bei der modernen Stadt den Eindruck, dass die Form hinreichend raffiniert und ausbalanciert genug ist, um bei einer einigermaßen sensiblen Verwendung allen denkbaren Ansprüchen gewachsen zu sein. Das soll nicht heißen, dass man aktuelle Tendenzen der Zersiedelung, der Segregation, der mangelnden Integration von Armen, Alten und Kindern, der Schrumpfung (mit der Entstehung von Leerräumen in einem durchaus buchstäblichen Sinn) nicht mit Besorgnis beobachten kann und muss (siehe nur Häußermann / Kronauer / Siebel 2004; Oswalt 2005; Oswalt / Rieniets 2006). Aber bereits diese Beobachtung ist ein Beleg dafür, dass die Form der Stadt nach wie vor funktioniert. Andernfalls würde nicht auffallen, welche Probleme sie gegenwärtig zu bewältigen hat. Ebenso wenig ist zu bestreiten, dass es nicht zahlreiche Möglichkeiten der weiteren Stadtentwicklung 
gibt, die noch uneingelöst sind, sich aber bereits als wünschenswert darstellen (siehe Reininghaus-Gesellschaft 2006; und vgl. von Borries / Böttger / Heilmeyer 2008). Aber auch das ist ein Beleg für die funktionierende, nach wie vor lebendige Form der modernen Stadt.

Dennoch ist über die Frage nachzudenken, ob sich mit der Einführung des Computers und seiner Derivate (Rechenzentren, Internet, Computernetzwerke) unter Umständen ein ähnlich tiefgehender Struktur- und Kulturumbruch der Gesellschaft ereignen wird oder aktuell bereits ereignet, wie er sich mit den Einführungen der Sprache, der Schrift und des Buchdrucks ereignet hat (vgl. Nora / Minc 1979; Castells 1996; Robertson 1998, 2003; Drucker 2003). Denn dann müssten wir vermuten, dass sich mit einer neuen Strukturform und einer neuen Kulturform der Gesellschaft auch die Anforderungen an ihre Stadt ändern und dass wir es mit der Frage zu tun bekommen, ob die genannten Probleme und Zielsetzungen der Stadtentwicklung bereits Teil dieser Umstellung sind oder von ihr ablenken.

Wieder ginge es um einen Medienumbruch, der ,das ganze psychische und soziale Gefüge (berührt)", in dem es sich der Mensch auf diesem Planeten einigermaßen heimisch gemacht hat (McLuhan 1994: 9). Und wieder wäre der Medienumbruch von einer Dimension, wie sie in einem Satz von Alfred North Whitehead ausgesprochen wird, den Marshall McLuhan und Quentin Fiore ihrem unter dem Titel „The Medium is the Massage“ erschienenen „inventory of effects" vorangestellt haben: „The major advances in civilization are processes that all but wreck the societies in which they occur" (McLuhan / Fiore 2001).

Wir können hier nur fragen, worin der Sinnüberschuss besteht, der mit der Einführung des Computers und seiner Derivate entsteht, welche Strukturform sich für die Sicherstellung der Verbreitung der Kommunikation im Medium des Computers andeutet, welche Kulturform den Sinnüberschuss selektiv bearbeitbar macht und, schließlich, welche Konsequenzen daraus und aus unseren bisherigen Überlegungen zur sozialen Form der Stadt für die Form der nächsten Stadt gezogen werden können. Wir verfügen über die Heuristiken der strukturellen Differenz und des leeren Platzes, um diese Form der nächsten Stadt weniger abzuleiten, denn mit einem Deduktionszusammenhang haben wir es hier nicht zu tun, als vielmehr einigermaßen zielsicher nach ihr zu suchen.

Der Sinnüberschuss der Kommunikation im Verbreitungsmedium des Computers, darin folgen wir Niklas Luhmann (1997: 302ff, 410ff), besteht darin, dass der Computer mit Gedächtnisleistungen und einer Schnelligkeit, die alles in der Kommunikation und im Bewusstsein bisher Erlebte übertrifft, Daten erarbeitet, verknüpft und auswertet, die wir bewusst und kommunikativ nicht nachvollziehen und kontrollieren und dennoch unserer Kommunikation zugrunde legen und in ihr verwenden müssen. Wir sprechen deswegen von einem Kontrollüberschuss der Kommunikation, von dem Wertpapierhändler an ihren Reuters-Bildschirmen, Ärzte mit ihren Diagnose-Unterstützungsgeräten, Ingenieure mit ihren Computer-Aided-Design-Programmen, Naturwissenschaftler mit ihren vernetzten Datenrechnern, Soldaten auf ihren informatisierten Schlachtfeldern und interaktive Medienkünstler einstweilen zwar mehr berichten können als der Normalverbraucher an seiner Suchmaschine, der aber dennoch in der gesamten Gesellschaft diesseits und deswegen, qua Exklusion, auch jenseits des digital divide zu spüren ist. Dieser Kontrollüberschuss bedeutet, dass wir uns in den Computern und mit den Computern auf Kommunikationen einlassen müssen, an denen die Computer durch ihre eigenen Programme kontrolliert auf eine Art und Weise teilnehmen, die wir unsererseits weder durchschauen noch kontrollieren können. In diesen Computern sind eigene Kontrollprojekte implementiert, die zwar, so wird vermutet, noch kein Eigenleben entwickelt haben, aber nicht mehr sehr weit davon entfernt sind.

Die Strukturform, auf die die Gesellschaft zurückgreift, um die Kommunikation im Verbreitungsmedium des Computers abzusichern, das heißt zu tragen und zu begrenzen zugleich, 
ist die Differenzierung der Gesellschaft nicht mehr in die funktionalen Systeme der modernen Gesellschaft, in die sozialen Schichten der antiken Hochkultur oder gar in die Stämme des Stammesgesellschaft, sondern in Netzwerke, die jedes für sich, gelingend oder misslingend, als ein Kontrollprojekt zu verstehen sind (vgl. je unterschiedlich Kelly 1990; White 1992; Deleuze 1993). Kontrollprojekte erkennt man daran, dass sie mit Elementen arbeiten, deren fragile, um nicht zu sagen labile Identität sie zu wechselnden, bei Bedarf robusten, aber auch wieder auflösbaren Verknüpfungen untereinander befähigt. Diese Identitäten sind heterogener Art. In der Regel handelt es sich um Kombinationen von Personen und Organisationen, Geschichten und Ideologien, Techniken und Orten, Organismen und Transzendenzen, um hybride Entitäten (Latour 1998), um flottierende Rhizome (Deleuze / Guattari 1992: 11ff), die sich unter Umständen so schnell wieder auflösen, wie sie sich gebildet haben, unter Umständen nur halten, so lange sie nicht thematisiert werden, und zuweilen aus nichts anderem bestehen als aus dem Reden über sie. Harrison C. White spricht mit Verweis auf eine zunehmende Festigkeit der Kopplung und Entkopplung von stories, disciplines, styles, regimes und hegemonies (White 2008).

Die Kulturform dieser nächsten Gesellschaft ist, so vermuten wir, die Form im Sinne des Begriffs Spencer-Browns. Nur deren Heuristik des Einschlusses des Ausgeschlossenen ist in der Lage, mit den komplexen Rekursionen von Kontrollprojekten umzugehen, in denen jede Entscheidung jetzt getroffen werden muss, obwohl und während alle Beteiligten sehen, dass man - nicht zuletzt wegen der an der Kommunikation beteiligten Computer - auch nicht andeutungsweise über vollständige, die Sache ausschöpfende Informationen verfügt. Hinreichende Informationen über unzureichende Informationen müssen genügen. ${ }^{15}$ Die Beobachtung der Form genügt diesen Anforderungen, weil sie es erlaubt, Markierungen zu setzen und zu bezeichnen, ohne eine mitlaufende und unbezeichnete Außenseite dabei aus den Augen zu verlieren. Die Beobachtung der Form ist nicht nur fehlerfreundlich, sondern auch ignoranzfreundlich (von Weizsäcker / von Weizsäcker 1984; Smithson 1989), insofern sie jeden Anspruch blockiert, einen Sach-, Zeit- oder Sozialverhalt expertenhaft auszubuchstabieren, und stattdessen Leerstellen bereithält, die es ihr erlauben, mit überraschenden Rückkopplungen jetzt schon zu rechnen.

Worin besteht der Platz für die Leere in der Stadt der nächsten Gesellschaft? Welche Handlungen, in der Moderne noch streng reguliert, werden in ihr freigegeben und können auch in ihr freigegeben werden, weil die Kommunikation längst neue Formen der Regulierung gefunden hat? Was wird in der Stadt der nächsten Gesellschaft beobachtbar, während anderes unbeobachtbar wird?

Gegenwärtig beansprucht die Kreativszene die größte Aufmerksamkeit, wenn es darum geht, neue und innovative Tendenzen der Stadtentwicklung auszumachen (Lange 2007). Aber diese Kreativszene ist nur die Spitze eines Eisbergs. Vermutlich geht es darüber hinaus, mit einem glücklichen Begriff von Michael Hardt und Antonio Negri in ihrem Buch Empire, ${ }^{16}$ um posses aller Art, die nicht nur künstlerisch motiviert sein müssen, sondern auch unternehmerisch und politisch, terroristisch und anarchistisch, sportlich und religiös motiviert sein können. Die posse, eine Bezeichnung sowohl für polizeiliche task forces als auch für Rap- und HiphopGruppen, besteht aus einer Handvoll Leute, die nicht zwingend gewalttätig, aber doch mit einer

15 Mit dieser Situation rechnet die Kybernetik, seit es sie gibt, siehe nur Ashby 1958; unüberbietbar genau auch der Titel eines Beitrags von John von Neumann: „Probabilistic Logics and the Synthesis of Reliable Organisms from Unreliable Components“" (von Neumann 1956).

16 Michael Hardt und Antonio Negri (2008: 408) lesen das Wort posse amerikanisch und lateinisch: als Verweis auf task forces und Rap-Gruppen und als Verweis auf potentia, das Vermögen, die Kraft, gleichsam ein Wissen, das auch die Fähigkeit der Durchsetzung hat, oder umgekehrt ein Wille zur Durchsetzung, der im Zuge der Durchsetzung auch das dafür erforderliche Wissen generiert. Siehe auch die Diskussion des Konzepts bei Müller (2008: 27ff). 
durchsetzungsstarken Kombination von Autonomie und Disziplin ein Projekt verfolgen, das zunächst einmal jede Unwahrscheinlichkeit auf seiner Seite hat (siehe auch den Begriff der „Komplizenschaft“ bei Ziemer 2007). Possen treten als Singularitäten auf, die vielfach vernetzt dennoch alles Wesentliche sich selber verdanken. Sie machen Märkte, Häuser und Pläne nicht überflüssig, im Gegenteil, sie durchkreuzen sie und beuten sie aus. Sie überlagern sich ihnen und greifen auf eine andere Logik zurück, die nicht mehr die Logik der Statuskonkurrenz, des strategischen Kalküls oder der funktionalen Ordnung ist, sondern eine Logik der Störung, des Zusammenbruchs (im Sinne der Heidegger-Lektüre von Winograd / Flores 1989: 267ff), die darauf zählt, dass sich nur so die Kontexte identifizieren lassen, auf die jede Neugestaltung angewiesen ist. Possen sind die Form, in der sich eine hyperkomplex gewordene Gesellschaft über sich selber informiert, auch wenn die alte funktionale Ordnung nur die Störung sieht und nicht die Neuordnung, die sich im Medium der Störung vollzieht.

Im Anschluss an den Markt der tribalen Stadt, die Häuser der antiken Stadt und die Pläne der modernen Stadt recodieren die Possen die Leerstellen der Stadt. Ihr literarisches Denkmal haben sie in Neal Stephensons Roman Snow Crash (1992) gefunden. Diese Leerstellen sind jetzt nicht mehr nur Gelegenheiten zum Tausch, Orte des strategischen Kalküls im Umgang mit der Gesellschaft und überraschende Ergebnisse einer funktionalen Planung der Stadt, sondern sie sind Anlässe für Hausbesetzungen, Straßenfeste, terroristische Angriffe, Fischgründe für Sekten, Standorte für unternehmerische Neugründungen, Gegenstand künstlerischer Aktionen, Bühne für politische Auftritte, die sich allesamt nicht mehr an repräsentative Räume halten, sondern sich ihre location je nach Situation, Anlass und Absicht selber suchen. Possen sind Singularitäten, die ihre wie immer ephemere, unter Umständen jedoch auch beachtlich belastbare Existenz mit einer großartig paradoxen Geste ausschließlich sich selber zu verdanken behaupten.

Das erste Opfer dieser Possen ist das Zentrum der Stadt. Es zu vermeiden beziehungsweise nur aufzusuchen, um es seiner Dezentralität zu überführen, ist nicht nur Ehrensache, sondern auch zwingender Bestandteil jeder Posse, weil anders ihre Singularität nur eine abgeleitete sein könnte, also keine wäre. Deswegen sind die Suburbs, die Siedlungsgebiete von Familienpossen, die ersten Formen dieser nächsten Stadt und sind die generischen, die eigenschaftslosen Städte, von denen Rem Koolhaas gesprochen hat (Koolhaas 1996), ihre zweite Form. In den Urwäldern von Diktaturen, in den Handelszonen (,offshore“) von im Übrigen staatsbürokratisch verwalteten Ländern, auf künstlichen Inseln vor erdölreichen Wüstenstaaten entstehen Städte, das ist ihre dritte Form, die als Possen ihrer selbst zu verstehen sind und zugleich daran erinnern, dass Städte in der gesamten Menschheitsgeschichte auf diese Weise als fürstliche oder kirchliche Gründung aus dem Boden gestampft werden konnten. Doch ist nicht auch die „usurpatorische conjuratio“, die in einem Eidschwur verbundene Gründung einer Stadt aus dem Willen der Herbeigelaufenen, von der Max Weber so angetan war, bereits eine Posse in diesem Sinne?

Wie dem auch sei, wichtig ist, dass das gesamte Repertoire der modernen Buchdruckgesellschaft, jedes Massenmedium, jedes ökonomische Kalkül, jede politische Taktik, jede künstlerische Aktion, jede religiöse Verbrämung, jeder rechtliche Trick, jede wissenschaftliche Rechtfertigung, jede urbane Strategie jetzt zum Ausgangspunkt, Zielpunkt oder Material einer Posse, einer Recodierung, eines kulturellen Hackings werden können (Düllo / Liebl 2005; vgl. als Vorbild die in der Zeitschrift l'internationale situationniste, Heft 1, 1958: bis 12, 1969, dokumentierten Ideen), die mit einer Unbekümmertheit von „strategischer Kommunikation“ sprechen und diese auch betreiben, als sei es vollkommen ausgeschlossen, dass der Begriff der Kommunikation eigens erfunden wurde, um gegen die Annahme der Kausalität der Welt etwas in der Hand zu haben und auf Prozesse loser Kopplung aufmerksam machen zu können (,il existe un tiers avant l'autre“, so Serres 1980: 85; vgl. Serres 1968). 
Und in der Tat könnte man Possen als Experimente betrachten, die auf Mischformen von Kausalität und Kommunikation rekurrieren, genauer: auf kreis-kausale Prozesse, die ihre Selbstorganisation aus ihrer Fähigkeit der Arbeit mit unzureichenden Anfängen, das heißt mit im Laufe des Prozesses rettend oder scheiternd aus den eigenen Erfahrungen generierten Informationen, gewinnen. Wenn dies allerdings, um unser hier maßgebendes Argument zu wiederholen, in so großem Stile kulturell freigegeben ist, wie wir es gegenwärtig in einer die Innovation und die Kreativität preisenden Gesellschaft beobachten können, dann muss die Gesellschaft Mittel und Wege gefunden haben, ihre strukturell unverzichtbare Kommunikation auf einer anderen Ebene zu verankern. Wenn die Kultur einer Gesellschaft auf Handlungen rekurriert, so unser Argument, die mit dem Kommunikationstyp der vorherigen Medienepoche experimentieren, dann bewegt sich die Struktur der Gesellschaft bereits auf der Ebene der nächsten Medienepoche.

Im Übrigen wird dieses Argument nicht dadurch eingeschränkt, dass inzwischen auch ein Großteil der Kommunikation im Medium des Computers, vor allem des Internets, in diesem Sinne freigegeben ist. Weder die umfangreiche Nutzung des Internets für die Kommunikation von Pornografie noch die Ausgestaltung des Internets für Zwecke der Publikation individueller, geschäftlicher und politischer Selbstdarstellung ändern daran etwas, dass das Verbreitungsmedium Computer seinen epochalen Unterschied als Rechenmaschine und nicht als Publikationsmaschine macht. Deswegen scheint mir auch die Vermutung irreführend zu sein, dass das World Wide Web spätestens im Modus Web 2.0 und dank der Einführung von Portalen des social networking bereits den nächsten Medienbruch markiert. Das Gegenteil ist der Fall. Die Freigabe des Computers für Zwecke der Publikation macht deutlich, dass auch daraus und gerade daraus wesentliche Daten gewonnen werden können, die ohne Interesse wären, hätten wir nicht den Computer, der sie für uns und für sich aufzubereiten vermag. ${ }^{17}$

Possen sind auch der tiefere Grund für den medialen Überraschungserfolg des Handys, das heißt einer mobilen Telekommunikation, die von manchen Beobachtern in ihren strukturellen und kulturellen Folgen für weiterreichend gehalten wird als die Einführung des Computers (siehe nur Katz / Aakhus 2002; Geser 2004; Kluth 2008). Handys erlauben es tendenziell jedem sozialen Zusammenhang, einem familiären genauso wie einem geschäftlichen, einem politischen genauso wie einem kriminellen, sich in eine Posse zu verwandeln, die ihre Energie nicht allein aus ihrem Kontakt, sondern ebenso aus der instantan und ubiquitär aufrufbaren Ausgrenzung aus der je aktuellen Umgebung bezieht. Das ändert jedoch nichts daran, dass hier der Vernetzungsmodus von Computern eher imitiert als übertroffen wird. Denn die Dislokation der Telekommunikation und die Einbettung ihres vormals eher intimen Charakters in eine breitere Öffentlichkeit, die zum Mithören gezwungen wird, ändern nichts daran, dass es hier um eine Kommunikation unter partiell Anwesenden geht, wie man sie auch früher schon kannte.

Wenn unsere Annahme der Ausdifferenzierung einer nächsten, auf der Kommunikation im Verbreitungsmedium des Computers beruhenden Gesellschaft zutrifft, dann haben wir es neben den beobachtbaren Kreativstädten mit einer unbeobachtbaren Stadt zu tun, die sich nicht auf den Austausch von Akten und Dokumenten beschränkt wie die moderne Gesellschaft, sondern Daten errechnet und verrechnet, die diese Akten und Dokumente und alle anderen mittlerweile auch multimedial verfassten Dateien (Fotos, Videos, Songs, Programme, Codes) einer eigenen Analyse unterzieht und nach eigenen Regeln neu kombiniert. Unbeobachtbar ist die Stadt der Metadaten und ihrer Algorithmen, solange nicht beide Textform annehmen und so ihrerseits zum Gegenstand von Metadaten und Algorithmen werden. Unbeobachtbar sind

17 Nicht die Benutzeroberfläche, sondern die hinter ihr arbeitenden Rechner machen „die Welt zur Google“, wie Peter Glaser in der Stuttgarter Zeitung vom 13. April 2005 so treffend, wenn auch nicht als erster, formuliert hat. 
die Informationen, die aus den Possen über die Possen produziert werden. Unbeobachtbar ist das Netzwerkkalkül, das die strukturelle Reintegration der differenzierten Gesellschaft herstellt und an keinem der städtischen Orte seinerseits einen Platz hat (siehe jedoch zur Rolle globaler Städte, die in der Lage sind, eine hinreichende Kontrolle zu artikulieren, Sassen 2004).

Ein weiteres Mal produziert die Stadt jene Unbekannten, die die Gesellschaft ihrer Struktur und Kultur schuldig zu sein glaubt, um gleich anschließend ihr Auskommen mit ihnen zu suchen. Innovative Possen innerhalb und außerhalb der so genannten Kreativszenen durchkreuzen eine Gesellschaft, die sich Rechenmaterial schafft, indem sie sich partiell, das heißt in Grenzen, unberechenbar macht. Possen treten auf und verschwinden wieder. Aber in ihnen, wie einst und immer noch auf Märkten, in Häusern, in Plänen, wird jene Intelligenz produziert, in der die Gesellschaft im Spiegel ihrer Städte ein Bild ihrer selbst erhält.

Können wir auch für die Stadt der nächsten Gesellschaft eine Größenordnung festhalten? Wenn wird uns wiederum an Bedingungen einer robusten Selbstreparatur orientieren, wie sie für die Stammesgesellschaft in der hinreichend großen, aber begrenzten Anzahl der Mitglieder liegen, für die Antike in der Befestigung der Stadt durch eine Mauer und in der Moderne in der Reichweite administrativer, an Bedingungen der demokratischen Partizipation rückgekoppelter Einheiten, können wir für die Stadt der nächsten Gesellschaft annehmen, dass die erforderliche Größenordnung der Stadt etwas mit ihrer Netzwerkfähigkeit zu tun hat, das heißt damit, in einem vermutlich globalen Zusammenhang sowohl attraktiv für Possen zu sein als auch über genügend Ressourcen zu verfügen, um Angriffe von Possen zu überstehen. Das ist keine an räumliche Grenzen (Anwesenheit, Befestigung, Territorium) gebundene anschauliche Größe, wie dies Saskia Sassen bei ihren Untersuchungen zur ,globalen Stadt“ ebenfalls feststellen konnte (Sassen 1991, 1994), doch es ist eine funktionale Größe, die von Raumplanern bei ihrer Untersuchung der Einbindung einer „Stadt“ in die globale Infrastruktur und der politischen, wirtschaftlichen, technischen und kulturellen Reparaturmechanismen, über die sie verfügt, präzise in Rechnung gestellt werden kann. Unter den Gesichtspunkten der räumlichen Ordnung einer Stadt wird man dann allerdings auch davon ausgehen können, dass sich auf einem bestimmten Territorium, wie es als moderne Verwaltungseinheit abgesteckt worden ist, mehrere Städte unter Einschluss mehrerer Leerräume selbst dort, wo Häuser für Büros und Wohnungen stehen, finden lassen, je nachdem, welche politischen, finanziellen, artistischen, religiösen oder wissenschaftlichen Szenen für welche globalen Bezüge attraktiv sind und auf welche globalen Ressourcen für ihre Reparatur zurückgreifen können. Aber auch jetzt wird sich die Stadt ebenso wenig wie zu Le Corbusiers Zeiten in Funktionsbereiche aufteilen lassen. Denn weder lassen sich die Possen entsprechend funktional zuweisen noch ist ein einzelner Funktionsbereich zu städtischen Reparaturen in der Lage (siehe Foner 2005). Deswegen sprechen wir von der Netzwerkfähigkeit der Stadt, mit der Konsequenz, eine städtische Einheit erst dann erkennen zu können, wenn sie den Beutezug einer Posse mit Erfolg überstanden hat.

Wenn man sich an die Soziologie hält (etwa an Latour 2001, 2007), ist erkennbar, dass die Leitidee der nächsten Gesellschaft nicht mehr der Kapitalbegriff der politischen Ökonomie ist, sondern der Umweltbegriff der, wenn man will, immer noch ,politischen“, weil immer noch Inklusion und Exklusion balancierenden Ökologie. Der Umweltbegriff ist präzise darauf eingestellt, das sowohl für den Organismus in seiner Nische als auch für den Beobachter in dessen Nische Unbeobachtbare zu beobachten, weil er lehrt, für den Organismus wie für den Beobachter mit blinden Flecken zu rechnen und daher jede Posse an der Grenze, die sie zieht und in Anspruch nimmt, stellvertretend für sie selbst und für ihre Umwelt zu erkennen.

Aus dieser Idee kann man eine Heuristik entwickeln, die möglicherweise auch für die Architektur und Stadtplanung der Städte der nächsten Gesellschaft brauchbar ist: Wie zieht man, wie baut man, wie schützt man Grenzen, wenn diese Grenzen weder mit Mauern noch mit offenen Räumen noch mit funktional bestimmten Arealen identisch sind und erst recht nicht 
an den Totems der frühen Menschheitsgeschichte erkannt werden können, sondern an Objekten, die vielfältig benutzbar sind, an gadgets, deren Gebrauchswert in den Verknüpfungen liegt, die sie nicht definieren, sondern attrahieren, ${ }^{18}$ an switches, die man umlegen kann, um sich im $\mathrm{Nu}$ in einer anderen Situation zu befinden?

Die Form der nächsten Stadt wird nur wenig mit der licht- und sauerstoffdurchfluteten funktionalen Ordnung des Wohnens, Arbeitens, sich Erholens und sich Bewegens zu tun haben, die die Botschaft der Charta von Athen definierte, mit der Le Corbusier und der Congrès International d'Architecture Moderne (CIAM) das Anforderungsprofil der modernen Stadt kurz vor ihrem historischen Ende auf den Punkt brachten, so sehr die Forderungen dieser Charta ihre Gültigkeit behalten haben (Hilpert 1984). Die Form der nächsten Stadt wird nicht mehr funktional, sondern konnektionistisch sein, allerdings konnektionistisch nicht in einem verträumt zivilgesellschaftlichen Sinne, so als ermögliche der Vernetzungsmodus des Computers endlich das Verschleifen aller Beteiligten in endlosen Rückkopplungen ihrer selbst, sondern in einem eher paradoxen Sinne der Knüpfung von Verbindungen durch ihre Kappung. Als wüsste Rem Koolhaas (er weiß es mit Sicherheit), dass endlose Rückkopplungen in chaotischem Rauschen enden, setzt er auf eine Idee, die selber eine Posse ist: auf die Idee der „Bigness“. Nur die Größe sei es, die Rückzug und Konzentration ermögliche, ,yielding the rest of a contested territory to enemy forces“" (Koolhaas 1998: 511).

Die nicht mehr funktionale, sondern konnektionistische Stadt setzt auf Possen, die die Singularität suchen, weil sie wissen, dass die Gesellschaft sich die Verknüpfung so oder so nicht nehmen lassen wird.

Die Stadt der nächsten Gesellschaft ist nicht extraterritorial wie die der Stammesgesellschaft, nicht institutionell wie die der antiken Hochkultur und nicht funktional wie die der modernen Gesellschaft, sondern sie ist innovativ und konnektionistisch. Sie setzt auf überraschende Verbindungen, auf Zufälle, die keine geheime, aber doch eine bis zu diesem Moment verborgene Logik offenbaren. Sie kann warten und sie kann beschleunigen. Sie kann Traditionen pflegen und sich im Handumdrehen von ihnen verabschieden. Sie erlaubt das Spiel, aber sie erwartet auch die Dokumentation. Analysten und Profiler werden ihre wichtigsten Experten, weil es bei all den Possen, die sie durchkreuzen, darauf ankommt, ihre Spur nicht zu verlieren, aber auch, die Possen auf ihr Potential ebenso wie auf ihre Rekombinierbarkeit hin zu untersuchen.

Dank: Eine Studie im Rahmen des Next City Reininghaus Projekts der Zeppelin University, Friedrichshafen, im Auftrag der Asset One ImmobilienentwicklungsAG, Graz. Für wichtige Hinweise danke ich Roland Koppensteiner, Andreas Kleboth und Max Rieder, Graz, Bernhard Krusche, Tübingen, Bastian Lange, Berlin, und Werner Baecker, Köln.

\section{Literatur}

Agnew, Jean-Christophe (1986): Worlds Apart: The Market and the Theater in Anglo-American Thought, 1550-1750, Cambridge.

Aristoteles (1970): Metaphysik: Schriften zur Ersten Philosophie, übersetzt und hrsg. von Franz F. Schwarz, Stuttgart.

18 Ich danke Tom Fürstner für seine Hinweise auf Max / MSP, ein Programm zur Codierung interaktiver Installationen, und auf Olinda, kein einfaches digitales Radio, sondern eine Pionierstudie in Sachen „social hardware“. Siehe http://www.cycling74.com/ und http://schulzeandwebb.com/2008/olinda/. Siehe auch die Versammlung der Bastler, Hacker und Spieler auf der seit 2006 jährlich stattfindenden Maker Fair, http://www.makerfaire.com/. 
Aristoteles (1981): Politik, übersetzt und mit erklärenden Anmerkungen versehen von Eugen Rolfes, mit einer Einleitung von Günther Bien, Hamburg.

Ashby, W. Ross (1958): Requisite Variety and Its Implications for the Control of Complex Systems, in: Cybernetica 1, S. 83-99.

Backhaus, Hans-Georg (1997): Dialektik der Wertform: Untersuchungen zur marxschen Ökonomiekritik, Freiburg.

Baecker, Dirk (2004 a): Platon oder die Form der Stadt, in: Ders., Wozu Soziologie? Berlin, S. 189-212.

Baecker, Dirk (2004 b): Miteinander leben, ohne sich zu kennen: Die Ökologie der Stadt, in: Soziale Systeme: Zeitschrift für soziologische Theorie 10, S. 257-272.

Baecker, Dirk (2004 c): Niklas Luhmann in der Gesellschaft der Computer, in: Ders., Wozu Soziologie?, Berlin, S. 125-149.

Baecker, Dirk (2007 a): Studien zur nächsten Gesellschaft, Frankfurt / Main.

Baecker, Dirk (2007 b): Communication With Computers, or How Next Society Calls for an Understanding of Form, in: Soziale Systeme 13, S. 409-420.

Baecker, Dirk (2007 / 08): The Network Synthesis of Social Action, I: Toward a Sociological Theory of Next Society, \& II: Understanding Catjects, in: Cybernetics and Human Knowing 14, S. 9-42, und 15, S. 45-65.

Bahrdt, Hans Paul (1998): Die moderne Großstadt: Soziologische Überlegungen zum Städtebau, Neuaufl., Opladen.

Baudrillard, Jean (1972): Pour une critique de l'économie du signe, Paris.

Benevolo, Leonardo (2007): Die Geschichte der Stadt, aus dem Italienischen von Jürgen Humburg, 9. Aufl., Frankfurt / Main.

Benjamin, Walter (1961): Paris, die Hauptstadt des XIX. Jahrhunderts, in: Ders., Iluminationen: Ausgewählte Schriften, Frankfurt / Main, S. 185-200.

Benjamin, Walter (2001): Das Passagen-Werk, hrsg. von Rolf Tiedemann, Frankfurt / Main.

Berking, Helmuth / Martina Löw (2005): Wenn New York nicht Wanne-Eickel ist...: Über Städte als Wissensobjekt der Soziologie, in: Dies. (Hrsg.), Die Wirklichkeit der Städte, Soziale Welt Sonderband 16, Baden-Baden, S. 9-22.

Brunner, Otto (1950): Die alteuropäische „Ökonomik“, in: Zeitschrift für Nationalökonomik 13, S. 114-139.

Burdett, Rick / Deyan Sudjic (Hrsg.) (2007): The Endless City: The Urban Age Project by the London School of Economics and Deutsche Bank's Alfred Herrhausen Society, London.

Callon, Michel (1998): An Essay on Framing and Overflowing: Economic Externalities Revisited by Sociology, in: Ders. (Hrsg.), The Laws of the Markets, Oxford, S. 244-269.

Calvino, Italo (1985): Die unsichtbaren Städte, aus dem Italienischen von Heinz Riedt, München.

Campbell, Donald T. (1969): Variation and Selective Retention in Socio-Cultural Evolution, in: General Systems 14, S. 69-85.

Castells, Manuel (1996): The Rise of the Network Society, Oxford.

de Certeau, Michel (1988): Kunst des Handelns, aus dem Französischen von Robert Vouillé, Berlin.

de Coulanges, Numa Denis Fustel (1961): Der antike Staat: Studie über Kultus, Recht und Einrichtungen Griechenlands und Roms, aus dem Französischen von Paul Weiß, Nachdruck, Graz.

Deleuze, Gilles (1993): Postskriptum über die Kontrollgesellschaften, in: Ders., Unterhandlungen: 1972-1990, aus dem Französischen von Gustav Roßler, Frankfurt / Main, S. 254-262.

Deleuze, Gilles / Félix Guattari (1992): Tausend Plateaus: Kapitalismus und Schizophrenie, aus dem Französischen von Gabriele Ricke und Ronald Vouillé, Berlin.

Diener, Roger et al. (2006): Die Schweiz: Ein städtebauliches Porträt, 3 Bücher und eine Karte, Basel. 
Douglas, Mary (1989): A Typology of Cultures, in: Max Haller / Hans-Joachim Hoffmann-Nowotny / Wolfgang Zapf (Hrsg.), Kultur und Gesellschaft. Verhandlungen des 24. Deutschen Soziologentags, Zürich 1988, Frankfurt / Main, S. 85-97.

Drucker, Peter F. (2003): Managing in the Next Society, New York.

Düllo, Thomas / Franz Liebl (Hrsg.) (2005): Cultural Hacking: Kunst des Strategischen Handelns, Wien.

Dunbar, Robin (2004): The Human Story: A New History of Mankind's Evolution, London.

Durkheim, Emile (1988): Über soziale Arbeitsteilung: Studie über die Organisation höherer Gesellschaften, aus dem Französischen von Ludwig Schmidts, mit einer Einleitung von Niklas Luhmann, 2. Aufl., Frankfurt / Main.

Foner, Nancy (Hrsg.) (2005): Wounded City: The Social Effects of the Attack on the World Trade Center, New York.

Foster, George M. (1965): Peasant Society and the Image of Limited Good, in: American Anthropologist 67, S. 293-315.

Foster, George M. (1967): Tzintzuntzan: Mexican Peasants in a Changing World, Boston.

Foucault, Michel (1997): Die Archäologie des Wissens, Frankfurt / Main.

Freud, Sigmund (1994): Das Unbehagen in der Kultur, in: Ders., Das Unbehagen in der Kultur und andere kulturtheoretische Schriften, Frankfurt / Main, S. 29-108.

Frey, William H. / Zachary Zimmer (2001): Defining the City, in: Ronan Paddison (Hrsg.), Handbook of Urban Studies, London, S. 15-35.

Geser, Hans (2004): Towards a Sociological Theory of the Mobile Phone, abgerufen im Mai 2004 unter http://socio.ch/mobile/t_geser1.pdf.

Goody, Jack / Ian Watt / Kathleen Gough (1981): Entstehung und Folgen der Schriftkultur, aus dem Englischen von Friedhelm Herborth, Frankfurt / Main.

Grierson, P. J. Hamilton (1980): The Silent Trade: A Contribution to the Early History of Human Intercourse, Reprint in: Dalton, George (Hrsg.), Research in Economic Anthropology: A Research Annual 3, S. 1-74.

Habermas, Jürgen (1990): Strukturwandel der Öffentlichkeit: Untersuchungen zu einer Kategorie der bürgerlichen Gesellschaft, Neuaufl., Frankfurt / Main.

Hardt, Michael / Antonio Negri (2000): Empire, Cambridge / Mass.

Häußermann, Hartmut / Martin Kronauer / Walter Siebel (Hrsg.) (2004): An den Rändern der Städte: Armut und Ausgrenzung, Frankfurt / Main.

Heimer, Carol A. (1992): Doing Your Job and Helping Your Friends: Universalistic Norms about Obligations to Particular Others in Networks, in: Nitin Nohria / Robert G. Eccles (Hrsg.), Networks and Organizations: Structure, Form, and Action, Boston / Mass., S. 143-164.

Herlyn, Ulfert (2004): Zum Bedeutungswandel der öffentlichen Sphäre: Anmerkungen zur Urbanitätstheorie von H. P. Bahrdt, in: Walter Siebel (Hrsg.), Die europäische Stadt, Frankfurt / Main, S. 212-130.

Hilpert, Thilo (1984): Le Corbusiers „Charta von Athen“: Texte und Dokumente, kritische Neuausgabe, Braunschweig.

Horkheimer, Max / Theodor W. Adorno (1969): Dialektik der Aufklärung: Philosophische Fragmente, Frankfurt / Main.

Jacobs, Carol (2008): Subversions of the Political: Plato's Republic, in: Dies., Skirting the Ethical, Stanford / CA, S. 45-110.

Kant, Immanuel (1964): Beantwortung der Frage: Was ist Aufklärung? In: Werke, Bd. XI, hrsg. von Wilhelm Weischedel, Frankfurt / Main, S. 53-61.

Katz, James E. / Mark A. Aakhus (Hrsg.) (2002): Perpetual Contact: Mobile Communication, Private Talk, Public Performance, Cambridge. 
Kelly, Kevin (1990): Out of Control: The New Biology of Machines, Social Systems, and the Economic World, Redwood City / Cal.

Kluth, Andreas (2008): Nomads at Last: A Special Report, in: The Economist, April 10th.

Koolhaas, Rem (1996): Die Stadt ohne Eigenschaften, in: arch+132, S. 21-27.

Koolhaas, Rem (1998): Bigness, in: Jennifer Siegler (Hrsg.), Small, Medium, Large, Extra-Large: Office for Metropolitan Architecture, Rem Koolhaas and Bruce Mau, 2. Aufl., New York, S. 495-516.

Kripke, Saul A. (1980): Naming and Necessity, Oxford.

Lacan, Jacques (1978): Psychoanalyse et cybernétique, ou de la nature du langage, in: Le Séminaire de Jacques Lacan: Texte établi par Jacques-Alain Miller, Livre II: Le moi dans la théorie de Freud et dans la technique de la psychoanalyse, 1954-1955, Paris, S. 339-354.

Lange, Bastian (2007): Die Räume der Kreativszenen: Cultrepreneurs und ihre Orte in Berlin, Bielefeld.

Latour, Bruno (1998): Wir sind nie modern gewesen: Versuch einer symmetrischen Anthropologie, aus dem Französischen von Gustav Roßler, Frankfurt / Main.

Latour, Bruno (2001): Das Parlament der Dinge: Für eine politische Ökologie, aus dem Französischen von Gustav Roßler, Frankfurt / Main.

Latour, Bruno (2007): Eine neue Soziologie für eine neue Gesellschaft: Einführung in die Akteur-Netzwerk-Theorie, aus dem Französischen von Gustav Roßler, Frankfurt / Main.

Le Corbusier (1963): Ausblick auf eine Architektur (Vers une achitecture), Berlin.

Leach, Edmund (1979): Kultur und Kommunikation: Zur Logik symbolischer Zusammenhänge, aus dem Englischen von Eberhard Bubser, Frankfurt / Main.

Ledda, Gavino (1980): Padre Padrone, aus dem Italienischen von Heinz Riedt, Frankfurt / Main.

Lefèbvre, Henri (1976): Die Revolution der Städte, aus dem Französischen von Ulrike Roeckl, Nachdruck, Frankfurt / Main.

Lévi-Strauss, Claude (1978): Strukturale Anhropologie, Bd 1, aus dem Französischen von Hans Naumann, Frankfurt / Main.

Lindner, Rolf (2005): Urban Anthropology, in: Helmuth Berking / Martina Löw (Hrsg.), Die Wirklichkeit der Städte, Soziale Welt Sonderband 16, Baden-Baden, S. 55-66.

Löw, Martina (2008): Soziologie der Städte, Frankfurt / Main.

Luhmann, Niklas (1997): Die Gesellschaft der Gesellschaft, Frankfurt / Main.

Lynch, Kevin (2007): Das Bild der Stadt, aus dem Amerikanischen von Henni Korsakoff-Schröder und Richard Michael, 5. Aufl., Basel.

Malinowski, Bronislaw (2005): Eine wissenschaftliche Theorie der Kultur, in: Ders., Eine wissenschaftliche Theorie der Kultur und andere Aufsätze, aus dem Englischen von Fritz Levi, Frankfurt / Main, S. 45-172.

Marcuse, Peter (2005): The Partitioning of Cities, in: Helmuth Berking / Martina Löw (Hrsg.), Die Wirklichkeit der Städte, Soziale Welt Sonderband 16, Baden-Baden, S. 257-276.

Marx, Karl (1980): Das Kapital: Kritik der politischen Ökonomie, 1. Bd, Berlin.

McLuhan, Marshal (1994): Die magischen Kanäle, aus dem Englischen von Meinrad Amann, Nachdruck, Dresden.

McLuhan, Marshall, Quentin Fiore (2001): The Medium is the Massage: An Inventory of Effects, Nachdruck, Corte Madera / Cal.

Mitchell, Sandra (2008): Komplexitäten: Warum wir erst anfangen, die Welt zu verstehen, aus dem Englischen von Sebastian Vogel, Frankfurt / Main.

Müller, Gini (2008): Possen des Performativen: Theater, Aktivismus und queere Politiken, Wien.

Nora, Simon / Alain Minc (1979): Die Informatisierung der Gesellschaft, dt. Frankfurt / Main. 
Ong, Walter J. (1987): Oralität und Literalität: Die Technologisierung des Wortes, aus dem Amerikanischen von Wolfgang Schömel, Opladen.

Oswalt, Philipp (Hrsg.) (2005): Schrumpfende Städte, 2 Bde, Ostfildern.

Oswalt, Philipp / Tim Rieniets (Hrsg.) (2006): Atlas der schrumpfenden Städte, Ostfildern.

Park, Robert E. (1967): The City: Suggestions for the Investigation of Human Behavior in the Urban Environment, in: Robert E. Park / Ernest W. Burgess / Roderick D. McKenzie, The City, Nachdruck mit einer Einführung von Morris Janowitz, Chicago, S. 1-46.

Platon (2000): Politeia, Sämtliche Werke. Übers. von Friedrich Schleiermacher, neu hrsg. von Ursula Wolf, Bd 2, 2. Aufl., Reinbek / Hamburg, S. 195-537.

Polanyi, Karl (1979): Aristoteles entdeckt die Volkswirtschaft, in: Ders., Ökonomie und Gesellschaft, mit einer Einleitung von S. C. Humphreys, aus dem Englischen von Heinrich Jelinek, Frankfurt / Main, S. 149-185.

Polanyi, Michael (1985): Implizites Wissen, aus dem Englischen von Horst Brühmann, Frankfurt / Main.

Reininghaus-Gesellschaft (Hrsg.) (2006): Konzeption des Wünschenswerten: Was Städte über die Zukunft wissen sollten, Wien.

Robertson, Douglas S. (1998): The New Renaissance: Computers and the Next Level of Civilization, New York.

Robertson, Douglas S. (2003): Phase Change: The Computer Revolution in Science and Mathematics, New York.

Sassen, Saskia (1991): The Global City: New York - London - Tokyo - Princeton / NJ.

Sassen, Saskia (1994): Cities in a World Economy: Sociology for a New Century, Thousand Oaks / Cal.

Sassen, Saskia (2004): Die Verflechtungen unter der Oberfläche der fragmentierten Stadt, in: Walter Siebel (Hrsg.), Die europäische Stadt, Frankfurt / Main, S. 373-384.

Serres, Michel (1968): Hermès I: La Communication, Paris.

Serres, Michel (1980): Le parasite, Paris.

Serres, Michel (1981): Der Parasit, aus dem Französischen von Michael Bischoff, Frankfurt / Main.

Siebel, Walter (2000): Urbanität, in: Hartmut Häußermann (Hrsg.), Großstadt: Soziologische Stichworte, 2. Aufl., Opladen, S. 264-272.

Siebel, Walter (2004): Einleitung: Die europäische Stadt, in: Ders. (Hrsg.), Die europäische Stadt, Frankfurt / Main, S. 11-50.

Sieferle, Rolf Peter (1997): Rückblick auf die Natur: Eine Geschichte des Menschen und seiner Umwelt, München.

Sieverts, Thomas (1999): Zwischenstadt: Zwischen Ort und Welt, Raum und Zeit, Stadt und Land, 3., verb. und um ein Nachwort erg. Aufl., Basel.

Simmel, Georg (1995): Die Großstädte und das Geistesleben, in: Gesamtausgabe, Bd. 7: Aufsätze und Abhandlungen 1901-1908, Bd. I, Frankfurt / Main, S. 116-131.

Smithson, Michael (1989): Ignorance and Uncertainty: Emerging Paradigms, New York.

Sohn-Rethel, Alfred (1978): Warenform und Denkform, mit zwei Anhängen, Frankfurt / Main.

Spencer-Brown, George (1997): Laws of Form: Gesetze der Form, aus dem Englischen von Thomas Wolf, Lübeck.

Stephenson, Neal (1992): Snow Crash, New York.

Stichweh, Rudolf (1992): Der Fremde - Zur Evolution der Weltgesellschaft, in: Rechtshistorisches Journal 11, S. 295-316.

Stichweh, Rudolf (1994): Raum, Region und Stadt in der Systemtheorie, in: Soziale Systeme: Zeitschrift für soziologische Theorie 4, S. 341-358. 
Stinchcombe, Arthur L. (2001): When Formality Works: Authority and Abstraction in Law and Organizations, Chicago.

Sudjic, Deyan (2007): Theory, Policy and Practice, in: Burdett, Rick / Deyan Sudjic (Hrsg.), The Endless City: The Urban Age Project by the London School of Economics and Deutsche Bank's Alfred Herrhausen Society, London, S. 32-51.

Tilly, Charles (1992): Coercion, Capital, and European States, AD 990-1992, Cambridge / Mass.

Turner, Victor (1969): The Ritual Process, Chicago.

Vernant, Jean-Pierre (1982): Die Entstehung des griechischen Denkens, aus dem Französischen von Edmund Jacoby, Frankfurt / Main.

von Borries, Friedrich / Matthias Böttger / Florian Heilmeyer (2008): Bessere Zukunft? Auf der Suche nach den Räumen von Morgen, Berlin.

von Neumann, John (1956): Probabilistic Logics and the Synthesis of Reliable Organisms from Unreliable Components, in: Claude E. Shannon / John McCarthy (Hrsg.), Automata Studies, Princeton / NJ, S. 43-98.

von Weizsäcker, Ernst Ulrich, und Christine von Weizsäcker (1984): Fehlerfreundlichkeit, in: Klaus Kornwachs (Hrsg.), Offenheit - Zeitlichkeit - Komplexität: Zur Theorie der Offenen Systeme, Frankfurt / Main, S. 167-200.

Weaver, Warren (1948): Science and Complexity, in: American Scientist 36, S. 536-544.

Weber, Max (1990): Wirtschaft und Gesellschaft: Grundriß der verstehenden Soziologie, Studienausgabe Tübingen.

Weber, Max (1991): Wirtschaftsgeschichte: Abriß der universalen Sozial- und Wirtschaftsgeschichte, 5. Aufl., Berlin.

White, Harrison C. (1992): Identity and Control: A Structural Theory of Action, Princeton / NJ.

White, Harrison C. (1995): Network Switches and Bayesian Forks: Reconstructing the Social and the Behavioral Sciences, in: Social Research 62, S. 1035-1063.

White, Harrison C. (2008): Identity and Control: How Social Formations Emerge, Princeton / NJ.

Winograd, Terry / Fernando Flores (1989): Erkenntnis Maschinen Verstehen: Zur Neugestaltung von Computersystemen, aus dem Amerikanischen von Ludwig Voet, Berlin.

Xenophon (1956): Oikonomikos: Die Hauswirtschaftslehre, in: Ders., Die sokratischen Schriften, übertragen und herausgeben von Ernst Bux, Stuttgart, S. 235-302.

Ziemer, Gesa (2007): Komplizenschaft: Eine Taktik und Ästhetik der Kritik?, in: Jörg Huber / Philipp Stoellger / Gesa Ziemer / Simon Zumsteg (Hrsg.), Ästhetik der Kritik: Verdeckte Ermittlung, Zürich, S. $75-81$.

Prof. Dr. Dirk Baecker

Zeppelin Universität

Lehrstuhl für Kulturtheorie und Kulturanalyse

Am Seemooser Horn 20

88045 Friedrichshafen

dirk.baecker@zeppelin-university.de 